\title{
A VARIATIONAL METHOD IN IMAGE RECOVERY*
}

\author{
GILLES AUBERT ${ }^{\dagger}$ AND LUMINITA VESE ${ }^{\ddagger}$
}

\begin{abstract}
This paper is concerned with a classical denoising and deblurring problem in image recovery. Our approach is based on a variational method. By using the Legendre-Fenchel transform, we show how the nonquadratic criterion to be minimized can be split into a sequence of half-quadratic problems easier to solve numerically. First we prove an existence and uniqueness result, and then we describe the algorithm for computing the solution and we give a proof of convergence. Finally, we present some experimental results for synthetic and real images.
\end{abstract}

Key words. image processing, Legendre-Fenchel transform, partial differential equations, calculus of variations

AMS subject classifications. 35J, 49J, 65N

PII. S003614299529230X

1. Introduction. An important problem in image analysis is the reconstruction of an original image $f$ describing a real scene from an observed image $p$. The transformation (or degradation) connecting $f$ to $p$ is in general the result of two phenomena. The first phenomenon is deterministic and is related to the mode of image acquisition (for example, the computation of integral projections in tomography) or to possible defects of the imaging system (blur created by a wrong lens adjustement, by a movement,...). The second phenomenon is random: the noise inherent degradation in any signal transmission. Suppose that the noise denoted by $\eta$ is white, Gaussian, and additive.

The simplest model accounting for both blur and noise is the linear degradation model: we suppose that $f$ is connected to $p$ by an equation of the form

$$
p=R f+\eta,
$$

where $R$ is a linear operator. (We remain, for the moment, intentionally vague on the exact significance of (1.1) - in particular, on the space on which this equation is defined.)

The reconstruction problem of $f$ can be identified, in that way, with an inverse problem: find $f$, from (1.1). In general, this problem is ill-posed in the sense of Hadamard. The information provided by $p$ and the model (1.1) is not sufficient to ensure the existence, uniqueness, and stability of a solution $f$.

It is therefore necessary to regularize the problem by adding an a priori constraint on the solution. The most classical and frequent approach in image reconstruction is a stochastic approach based, in the framework of Bayesian estimation, on the use of maximum a posteriori (MAP) estimation. Supposing that $f$ is a Markov field (which constitutes an a priori constraint), then the MAP criterion identifies with a minimization problem in which the energy $J$ depends on the image $f$ and on the gradient. We are not going into the details of this approach; instead, we refer the

${ }^{*}$ Received by the editors September 22, 1995; accepted for publication (in revised form) February 6,1996 .

http://www.siam.org/journals/sinum/34-5/29230.html

†Laboratoire de Mathématiques, Universite de Nice, Parc Valrose, BP 71, F 06108 Nice, Cedex 02, France (gaubert@math.unice.fr).

${ }^{\ddagger}$ Laboratoire de Mathématiques, Universite de Nice, Parc Valrose, BP 71, F 06108 Nice, Cedex 02, France (luminita@math.unice.fr). 
reader to the original article of Geman and Geman [13] or, for a clear and synthetic exposition, to the work of Charbonnier [4].

Our purpose being to study the problem of image reconstruction via the calculus of variations and partial differential equations, we do not develop a new model here. We will study for a continuous image the model described by Geman and Geman for a numerical image (or a slight modification of this).

In section 2, we will present more precisely the minimization problem studied here, as well as the assumptions to impose on the model. These will be imposed by the requirements that we wish to obtain on the results. In section 3 we will show by using the Fenchel-Legendre transform how we can introduce in the energy $J$ (possibly nonconvex) a dual variable $b$ allowing us to reduce the minimization of $J$ to a sequence of quadratic minimization problems. In section 4 we will study the problem of the existence and uniqueness of a solution $f$. The obtained results are based on a singular perturbation result of Temam [12]. Moreover, we note the analogy of our problem with the one of minimal surfaces studied by Temam in [12] and [26]. In section 5 we will describe the algorithm in continuous variables for computing the solution, as well as a convergence proof. Finally, in sections 6 and 7, we develop the numerical analysis of the approximated problem, and we try to validate the model by presenting some examples with synthetic or real images.

2. Description of the model. Assumptions. In continuous variables the observed image $p$ and the reconstructed image $f$ can be represented by functions of $\Omega \subset$ $\mathbb{R}^{2} \rightarrow \mathbb{R}$ which associate with the pixel $(x, y) \in \mathbb{R}^{2}$ its gray level $p(x, y)$ or $f(x, y) ; \Omega$ is the support of the image (a rectangle in general). The gray levels being in finite number, we can suppose that the observation $p(x, y)$ verifies $0 \leq p(x, y) \leq 1 \forall(x, y) \in \Omega$.

The stochastic model proposed by Geman and Geman for image reconstruction leads us, as we pointed out in the introduction, to search for a solution among the minima of the energy

$$
J_{\alpha}(f)=\int_{\Omega}(p(x, y)-(R f)(x, y))^{2} d x d y+\alpha \int_{\Omega} \phi(|D f(x, y)|) d x d y .
$$

$R$ is a linear operator of $L^{2}(\Omega) \rightarrow L^{2}(\Omega)$, and the first integral represents an attached term on the data. The function $\phi: \mathbb{R}^{+} \rightarrow \mathbb{R}^{+}$is to be defined and symbolizes the regularization term (hence, a constraint on the solution). In [13], Geman and Geman have added a regularization term of the form

$$
\int_{\Omega}\left(\phi\left(\frac{\partial f}{\partial x}\right)+\phi\left(\frac{\partial f}{\partial y}\right)\right) d x d y
$$

corresponding (for numerical images) to a regularization on lines and columns. This term, unlike ours, is not invariant under rotation. The number $\alpha \in \mathbb{R}^{+}$is a parameter which allows us to balance the influence of each integral in the energy $J_{\alpha}(f)$. If $\alpha=0$, $J_{\alpha}(f)$ is

$$
J_{0}(f)=\int_{\Omega}(p(x, y)-(R f)(x, y))^{2} d x d y,
$$

and so the energy is reduced only to the attached term on the data, and the problem

$$
\inf _{f} J_{0}(f)
$$


corresponds to the least-squares method associated with the equation (1.1). Formally, every solution of (2.2) verifies the equation

$$
R^{*} p=R^{*} R f
$$

where we have denoted by $R^{*}$ the adjoint operator of $R$. Generally, (2.3) is an ill-posed problem: $R^{*} R$ is not always invertible or the problem (2.3) is often unstable.

To overcome this difficulty, we either look for a solution in a smaller set (where we have some compactness), or we add a regularization term to the attached term on the data. This method, which is due to Tikhonov [27], is the one we use here, and the additional term is represented in $(2.1)$ by $\int_{\Omega} \phi(|D f(x, y)|) d x d y$. It now remains to find some appropriate conditions on the function $\phi$ in order to satisfy the following principle of image analysis:

The reconstructed image must be formed by homogeneous regions, separated by sharp edges.

The model must, therefore, diffuse within the regions where the variations of gray levels are weak, and otherwise, it must preserve the boundaries of these regions; that is, it must respect the strong variations of gray levels.

So, supposing that the integrals in $J_{\alpha}(f)$ have made sense, then any function realizing the minima of $J_{\alpha}$ must formally verify (for instance, in the sense of distribution) the Euler equation $J_{\alpha}^{\prime}(f)=0$ or

$$
-\frac{\alpha}{2} \operatorname{div}\left(\frac{\phi^{\prime}(|D f|)}{|D f|} D f\right)+R^{*} R f=R^{*} p .
$$

Writing (2.5) in a nonconservative form, we will obtain some sufficient assumptions on $\phi$, in order to respect, as much as possible, the principle (2.4). To do this, for each point $(x, y)$ where $|D f(x, y)| \neq 0$, let the vectors $T(x, y)=\frac{D f(x, y)}{|D f(x, y)|}$ in the gradient direction, and $\xi(x, y)$ in the orthogonal direction to $T(x, y)$. With the usual notations $f_{x}, f_{y}, f_{x x}, \ldots$ for the first and second partial derivatives of $f$, and by formally developing the divergence operator, (2.5) can be written as

$$
-\frac{\alpha}{2}\left(\frac{\phi^{\prime}(|D f|)}{|D f|}\right) f_{\xi \xi}-\frac{\alpha}{2} \phi^{\prime \prime}(|D f|) f_{T T}+R^{*} R f=R^{*} p,
$$

where we have denoted by $f_{\xi \xi}$ and $f_{T T}$ the second derivatives of $f$ in the directions $\xi(x, y)$ and $T(x, y)$, respectively:

$$
\begin{aligned}
f_{\xi \xi} & =\frac{1}{|D f|^{2}}\left(f_{x}^{2} f_{y y}+f_{y}^{2} f_{x x}-2 f_{x} f_{y} f_{x y}\right), \\
f_{T T} & =\frac{1}{|D f|^{2}}\left(f_{x}^{2} f_{x x}+f_{y}^{2} f_{y y}+2 f_{x} f_{y} f_{x y}\right) .
\end{aligned}
$$

If $f$ is regular (at least continuous), we can interpret the principle (2.4) in the following manner (as shown in Figure 2.1): locally, we represent a contour $\mathcal{C}$ separating two homogeneous regions of the image, by a level curve of $f: \mathcal{C}=\{(x, y) ; f(x, y)=c\}$. In this case, the vector $T(x, y)$ is normal to $\mathcal{C}$ at $(x, y) \in \mathcal{C}$, and the expression $\frac{f_{\xi \xi}(x, y)}{|D f(x, y)|}=\operatorname{div}\left(\frac{D f(x, y)}{|D f(x, y)|}\right)$ represents the curvature of $\mathcal{C}$ at this point. 


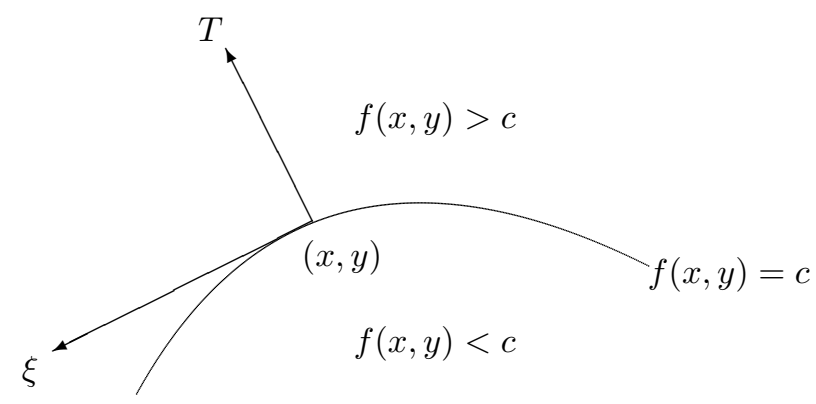

FIG. 2.1. A contour $\mathcal{C}$ separating two homogeneous regions.

In the interior of the homogeneous regions

$$
\{(x, y), f(x, y)<c\} \bigcup\{(x, y), f(x, y)>c\},
$$

where the variations of $f$ are weak, we want to encourage smoothing. If we suppose that $\phi^{\prime \prime}$ exists, with

$$
\phi^{\prime}(0)=0 \text { and } \phi^{\prime \prime}(0)>0,
$$

we obtain that in a neighborhood of $t=0,(2.6)$ is formally

$$
-\frac{\alpha}{2} \phi^{\prime \prime}(0)\left(f_{\xi \xi}+f_{T T}\right)+R^{*} R f=R^{*} f,
$$

and since for any orthogonal directions $T$ and $\xi$ we always have

$$
f_{\xi \xi}+f_{T T}=f_{x x}+f_{y y}=\triangle f,
$$

(2.8) is written as

$$
-\frac{\alpha}{2} \phi^{\prime \prime}(0) \triangle f+R^{*} R f=R^{*} p .
$$

Therefore, at the points where the image has a weak gradient, $f$ is a solution of (2.9), which is a uniformly elliptic equation having (this is well known) strong regularization diffusion properties on the solution.

On the contrary, in a neighborhood of a contour $\mathcal{C}$, the image presents a strong gradient. If we wish to better preserve this contour, it is preferable to diffuse only in the direction parallel to $\mathcal{C}$, i.e., in the $\xi$-direction. For this, it will be sufficient in (2.6) to annihilate, for strong gradients, the coefficient of $f_{T T}$ and to suppose that the coefficient of $f_{\xi \xi}$ does not vanish:

$$
\begin{array}{r}
\lim _{t \rightarrow+\infty} \phi^{\prime \prime}(t)=0, \\
\lim _{t \rightarrow+\infty} \frac{\phi^{\prime}(t)}{t}=m>0 .
\end{array}
$$

This allows us to reduce (2.6) in a neigborhood of $+\infty$ to an equation of the form

$$
-\frac{\alpha}{2} m f_{\xi \xi}+R^{*} R f=R^{*} p
$$

which we can interpret as a regularizing equation in the $\xi$-direction. 
But (2.10) and (2.11) are not compatible, and one must make a compromise between these two hypotheses; for example, by supposing that $\phi^{\prime \prime}(t)$ and $\phi^{\prime}(t) / t$ both converge to zero as $t \rightarrow \infty$ but with different speeds. More precisely, we suppose that

$$
\begin{gathered}
\lim _{t \rightarrow+\infty} \phi^{\prime \prime}(t)=\lim _{t \rightarrow+\infty} \frac{\phi^{\prime}(t)}{t}=0, \\
\lim _{t \rightarrow+\infty} \frac{\phi^{\prime \prime}(t)}{\frac{\phi^{\prime}(t)}{t}}=0
\end{gathered}
$$

that is, that $\phi^{\prime \prime}$ converges faster to 0 than $\phi^{\prime}(t) / t$, which makes preponderant the coefficient of $f_{\xi \xi}$ in $(2.6)$.

The preceding assumptions, (2.7) and (2.12), are rather of qualitative type and represent a priori the properties that we want to obtain on the solution. But these are not sufficient to prove that the model is well posed mathematically. To do this, in order to use the direct method of calculus of variations, we suppose that

$$
\lim _{t \rightarrow+\infty} \phi(t)=+\infty
$$

this assumption ensures the boundness of the minimizing sequences of

$$
J_{\alpha}(f)=\int_{\Omega}(p-R f)^{2} d x d y+\alpha \int_{\Omega} \phi(|D f|) d x d y .
$$

This growth to infinity must not be too strong because it must not penalize strong gradients (or formation of edges). Hence, we suppose a linear growth to infinity:

$$
\left\{\begin{array}{c}
\text { There exist constants } a_{i}>0 \text { and } b_{i} \geq 0, i=1,2 \text {, such that } \\
a_{1} t-b_{1} \leq \phi(t) \leq a_{2} t+b_{2} \forall t \in \mathbb{R}^{+}
\end{array}\right.
$$

and then the natural space on which we seek the solution will be

$$
\mathcal{V}=\left\{f \in L^{2}(\Omega), D f \in L^{1}(\Omega)^{2}\right\}
$$

Finally, for passing to the limit on the minimizing sequences of (2.1) and to obtain the uniqueness of a solution, we suppose that

$$
t \rightarrow \phi(t) \text { is strictly convex on } \mathbb{R}^{+} \rightarrow \mathbb{R}^{+} .
$$

Remark. A better growth condition than (2.14), which doesn't penalize the formation of edges, could be $\lim _{t \rightarrow \infty} \phi(t)=c>0$. In this case, if $M$ denotes a minimal threshold representing strong gradients, then the contribution of the integral $\int_{|D f| \geq M} \phi(|D f|) d x d y$ in the energy is nearly a constant and then the formation of an edge does not "cost" anything in the energy. But the hypothesis of a horizontal asymptote introduces in general a nonconvexity on $\phi$ for $t \geq M$, and we know, in this case, that the problem is ill-posed and can have no solution. Nevertheless, we have done some numerical tests with the function $\phi(t)=\frac{t^{2}}{1+t^{2}}$ (which is of this type of potential and verifies (2.7) and (2.12a)). The results obtained are very satisfactory.

To clarify the exposition, we now summarize our assumptions on the potential $\phi$. 
Hypotheses for $\phi$.

(H1) The function $\phi: \mathbb{R}^{+} \rightarrow \mathbb{R}^{+}$is of class $C^{2}$, is nondecreasing, and satisfies $\phi^{\prime}(0)=0$ and $\phi^{\prime \prime}(0)>0$.

(H2) The function $\phi: \mathbb{R}^{+} \rightarrow \mathbb{R}^{+}$has the properties

$$
\lim _{t \rightarrow+\infty} \phi^{\prime \prime}(t)=\lim _{t \rightarrow+\infty} \frac{\phi^{\prime}(t)}{t}=0 \text { and } \lim _{t \rightarrow+\infty} \frac{\phi^{\prime \prime}(t)}{\frac{\phi^{\prime}(t)}{t}}=0 .
$$

(H3) There exist constants $a_{i}>0$ and $b_{i} \geq 0, i=1,2$, such that

$$
a_{1} t-b_{1} \leq \phi(t) \leq a_{2} t+b_{2} \forall t \in \mathbb{R}^{+} .
$$

(H4) The function $\phi: \mathbb{R}^{+} \rightarrow \mathbb{R}^{+}$is strictly convex.

If it will be necessary to define the function $\phi$ on the whole space, we will extend it by parity of $\mathbb{R}^{+}$to $\mathbb{R}$. Other hypotheses due to the numerical approximation will be added in the following sections.

Of course, there are many functions $\phi$ verifying (H1)-(H4), and no criterion permits the choice of a potential more than any other. Charbonnier, in [4], presents many choices used in image reconstruction as well as a comparative study. Our choice here for the tests is the function $\phi(t)=\sqrt{1+t^{2}}$ which verifies (H1)-(H4) and, moreover, has a simple and geometric interpretation (the problem of minimal surfaces).

This paper is closely related to the works of Malik and Perona [20], Catté et al. [2], Rudin and Osher [22], and Chambolle and Lions [3]. Our approach is more oriented towards the techniques of the calculus of variations than those of PDEs. This paper completes, in a theoretical point of view, a preceding work concerning tomographic reconstruction [5], [6]. See also [1], [14], [29].

3. Auxiliary variable. Half-quadratic reduction. Before proving the existence of a solution, we will show in this section how we can associate an auxiliary variable (or dual) with the image $f$ and how the regularization term in the energy (2.1) can be represented by the infimum of quadratic functions. We recall that the energy $J_{\alpha}(f)$ is

$$
J_{\alpha}(f)=\int_{\Omega}(p-R f)^{2} d x d y+\alpha \int_{\Omega} \phi(|D f|) d x d y,
$$

the regularization term being

$$
L_{\phi}(f)=\int_{\Omega} \phi(|D f|) d x d y .
$$

To develop this idea, we use the Fenchel-Legendre transform (see Rockafellar [21] or Ekeland and Temam [12]). We recall that if $l(\xi)$ is a convex function of $\mathbb{R}^{N}$ into $\mathbb{R}$, then its Fenchel-Legendre transform (or polar) is the convex function $l^{*}\left(\xi^{*}\right)$ defined by

$$
l^{*}\left(\xi^{*}\right)=\sup _{\xi \in \mathbb{R}^{N}}\left(\xi \cdot \xi^{*}-l(\xi)\right)
$$

( $\xi \cdot \xi^{*}$ is the usual scalar product). This definition can be extended, without difficulty, to infinite-dimensional spaces. Let $\Omega$ be an open set of $\mathbb{R}^{N}$ and $l$ a convex continuous function of $\mathbb{R}^{N} \rightarrow \mathbb{R}$, and for $u \in L^{\gamma}(\Omega)^{N}$, let the functional

$$
L(u)=\int_{\Omega} l(u(x)) d x .
$$


Then the polar of $L$, denoted $L^{*}$, is defined on $L^{\gamma^{\prime}}(\Omega)^{N}$, the dual space of $L^{\gamma}(\Omega)^{N}$, where $\frac{1}{\gamma}+\frac{1}{\gamma^{\prime}}=1$, by

$$
L^{*}\left(u^{*}\right)=\sup _{u \in L^{\gamma^{\prime}}(\Omega)^{N}}\left\{\int_{\Omega} u(x) u^{*}(x)-\int_{\Omega} l(u(x)) d x\right\} .
$$

If, in addition, $l$ is nonnegative or if $l$ verifies an inequality of the type $l(\xi) \geq a(x)-$ $b \cdot|\xi|_{\mathbb{R}^{N}}^{\eta}$, with $a(x) \in L^{1}(\Omega), b \geq 0$, and $\eta \in[1, \infty)$, and if there exists $u_{0} \in L^{\infty}(\Omega)^{N}$ such that $L\left(u_{0}\right)<\infty$, then we can prove (see Ekeland and Temam [12, Chap. IX]) that $L^{*}\left(u^{*}\right)$ is written as

$$
L^{*}\left(u^{*}\right)=\int_{\Omega} l^{*}\left(u^{*}(x)\right) d x .
$$

Of course, we can reiterate the process and define

$$
L^{* *}(u)=\int_{\Omega} l^{* *}(u(x)) d x
$$

Since $l$ is convex, we have $l(\xi)=l^{* *}(\xi)$ and then $L^{* *}(u)=L(u)$.

We use this notion of polarity in our problem with $N=2, \gamma=\gamma^{\prime}=2$. Let, for $\xi, \xi^{*} \in \mathbb{R}^{2}$,

$$
\begin{gathered}
l(\xi)=\frac{|\xi|^{2}}{2}-\phi(|\xi|), \\
\psi\left(\xi^{*}\right)=l^{*}\left(\xi^{*}\right)-\frac{\left|\xi^{*}\right|^{2}}{2},
\end{gathered}
$$

as well as the functionals defined on $L^{2}(\Omega)^{2}$ by

$$
\begin{array}{ll}
\Phi(u) & =\int_{\Omega} \phi(|u(x, y)|) d x d y \quad\left(=\int_{\Omega}\left(\frac{|u(x, y)|^{2}}{2}-l(u(x, y))\right) d x d y\right) \\
\Psi(b) & =\int_{\Omega} \psi(b(x, y)) d x d y
\end{array}
$$

The following theorem proves that $\Phi$ and $\Psi$ are dual in a certain sense.

THEOREM 3.1. If $\phi$ (extended by parity on $\mathbb{R}$ ) verifies the following hypotheses:

(H3) there exist constants $a_{i}>0$ and $b_{i} \geq 0, i=1,2$, such that

$$
a_{1}|t|-b_{1} \leq \phi(t) \leq a_{2}|t|+b_{2} \forall t \in \mathbb{R} ;
$$

(H5) the function $t \rightarrow \frac{t^{2}}{2}-\phi(t)$ is convex on $\mathbb{R}$,

$$
\begin{aligned}
& \Phi(u)=\inf _{b \in L^{2}(\Omega)^{2}} \int_{\Omega}\left(\frac{|u-b|^{2}}{2}+\psi(b)\right) d x d y, \\
& \Psi(b)=\sup _{u \in L^{2}(\Omega)^{2}} \int_{\Omega}\left(-\frac{|u-b|^{2}}{2}+\phi(|u|)\right) d x d y .
\end{aligned}
$$

Proof. We prove (3.7). Let $\rho(u)$ be the value of the infimum in (3.7):

$$
\rho(u)=\int_{\Omega} \frac{|u|^{2}}{2} d x d y+\inf _{b} \int_{\Omega}\left(\frac{|b|^{2}}{2}-b \cdot u+\psi(b)\right) d x d y .
$$


This can be also written, with (3.4), as

$$
\begin{aligned}
\rho(u) & =\int_{\Omega} \frac{|u|^{2}}{2} d x d y+\inf _{b} \int_{\Omega}\left(\frac{|b|^{2}}{2}-b \cdot u+l^{*}(b)-\frac{|b|^{2}}{2}\right) d x d y \\
& =\int_{\Omega} \frac{|u|^{2}}{2} d x d y-\sup _{b} \int_{\Omega}\left(b \cdot u-l^{*}(b)\right) d x d y .
\end{aligned}
$$

Then (by Ekeland and Temam [12]),

$$
\rho(u)=\int_{\Omega} \frac{|u|^{2}}{2} d x d y-\int_{\Omega} l^{* *}(u) d x d y .
$$

From (H5) we have that $l^{* *}(\xi)=l(\xi) \forall \xi \in \mathbb{R}^{2}$; hence

$$
\rho(u)=\int_{\Omega} \frac{|u|^{2}}{2} d x d y-\int_{\Omega} l(u) d x d y=\Phi(u) .
$$

Equation (3.8) can be proved in the same manner.

We have remarked that we must seek a solution on the space

$$
\mathcal{V}=\left\{f \in L^{2}(\Omega), D f \in L^{1}(\Omega)^{2}\right\} .
$$

But in order to use the duality, we will look for a solution $f$ on the space $H^{1}(\Omega)$.

By using the relation $(3.7), J_{\alpha}(f)$ is written, for $f \in H^{1}(\Omega)$, as

$$
J_{\alpha}(f)=\int_{\Omega}(p-R f)^{2} d x d y+\alpha \inf _{b \in L^{2}(\Omega)^{2}} \int_{\Omega}\left(\frac{|D f-b|^{2}}{2}+\psi(b)\right) d x d y,
$$

and then

$$
\inf _{f \in H^{1}(\Omega)} J_{\alpha}(f)=\inf _{f \in H^{1}(\Omega)} \inf _{b \in L^{2}(\Omega)^{2}}\left[\int_{\Omega}(p-R f)^{2} d x d y+\alpha \int_{\Omega}\left(\frac{|D f-b|^{2}}{2}+\psi(b)\right) d x d y\right] .
$$

Because we can always invert the infinima, we get

$$
\inf _{f \in H^{1}(\Omega)} J_{\alpha}(f)=\inf _{b \in L^{2}(\Omega)^{2}}\left[\alpha \int_{\Omega} \psi(b) d x d y+\inf _{f \in H^{1}(\Omega)} \int_{\Omega}\left((p-R f)^{2}+\alpha \frac{|D f-b|^{2}}{2}\right) d x d y\right]
$$

and the method is now clear: we fix $b \in L^{2}(\Omega)^{2}$ and we solve the problem

$$
\inf _{f \in H^{1}(\Omega)}\left[\int_{\Omega}\left((p-R f)^{2}+\alpha \frac{|D f-b|^{2}}{2}\right) d x d y\right] .
$$

If $R$ satisfies some appropriate assumptions, then $\left(\mathcal{P}_{b}\right)$ has a unique solution, which is, formally, a solution of the Euler equation:

$$
\begin{cases}-\frac{\alpha}{2} \triangle f_{b}+R^{*} R f=R^{*} p-\frac{\alpha}{2} \operatorname{div} b, & \text { in } \mathcal{D}^{\prime}(\Omega), \\ \frac{\partial f_{b}}{\partial \eta}=0, & \text { on } \partial \Omega .\end{cases}
$$

We have then, for all $v \in H^{1}(\Omega)$ and for all $b$,

$$
\int_{\Omega}\left(\left(p-R f_{b}\right)^{2}+\frac{\alpha}{2}\left|D f_{b}-b\right|^{2}\right) d x d y \leq \int_{\Omega}\left((p-R v)^{2}+\alpha \frac{|D v-b|^{2}}{2}\right) d x d y .
$$


By adding $\int_{\Omega} \psi(b) d x d y$ on each side of (3.10), and by passing to the infimum in $b$, we get, for all $v \in H^{1}(\Omega)$,

$$
\begin{array}{r}
\inf _{b \in L^{2}(\Omega)^{2}}\left[\int_{\Omega}\left(\left(p-R f_{b}\right)^{2}+\frac{\alpha}{2}\left|D f_{b}-b\right|^{2}+\alpha \psi(b)\right) d x d y\right. \\
\leq \int_{\Omega}\left((p-R v)^{2}+\alpha \phi(|D v|)\right) d x d y
\end{array}
$$

Denoting

$$
T(b)=\int_{\Omega}\left(\left(p-R f_{b}\right)^{2}+\frac{\alpha}{2}\left|D f_{b}-b\right|^{2}+\alpha \psi(b)\right) d x d y,
$$

it is then sufficient, in order to prove that our algorithm allows us to solve the initial reconstruction problem, to obtain the existence of $b_{0} \in L^{2}(\Omega)^{2}$ such that

$$
\begin{aligned}
& T\left(b_{0}\right)=\inf _{b \in L^{2}(\Omega)^{2}} T(b) \text { with } \\
& T\left(b_{0}\right)=\int_{\Omega}\left(\left(p-R f_{b_{0}}\right)^{2}+\alpha \phi\left(\left|D f_{b_{0}}\right|\right)\right) d x d y=J_{\alpha}\left(f_{b_{0}}\right) .
\end{aligned}
$$

We will then deduce, with (3.11), that

$$
J_{\alpha}\left(f_{b_{0}}\right) \leq J_{\alpha}(v) \quad \forall v \in H^{1}(\Omega) .
$$

At this stage we must precisely formulate the mathematical assumptions in order to ensure the existence and uniqueness of a solution. There is a problem due to the fact that we work with sets constructed from the nonreflexive Banach space $L^{1}(\Omega)$.

4. Existence and uniqueness of a solution. To simplify, we will suppose that $R=I$ on $L^{2}(\Omega)$ (which corresponds to a denoising problem), and we will indicate in Appendix $\mathrm{B}$ the minor modifications to add if $R \neq I$. We also suppose that the weighting parameter $\alpha$ is equal to 1 , which does not modify the theoretical study of the problem (its presence and adjustment are fundamental in the applications). The studied functional is therefore

$$
J(f)=\int_{\Omega}(p-f)^{2} d x d y+\int_{\Omega} \phi(|D f|) d x d y .
$$

The basic assumptions that we will suppose to be verified in this section are as follows:

$$
\begin{aligned}
& p \in L^{\infty}(\Omega) \text { and } 0 \leq p(x, y) \leq 1 \text { a.e. }(x, y) \in \Omega, \\
& \phi: \mathbb{R} \rightarrow \mathbb{R} \text { is even, of class } C^{2} \text {, nondecreasing on } \mathbb{R}^{+} \text {, and there exist constants } \\
& a_{i}>0, b_{i} \geq 0, i=1,2, \text { such that } a_{1}|t|-b_{1} \leq \phi(t) \leq a_{2}|t|+b_{2} \forall t \in \mathbb{R},
\end{aligned}
$$

$$
0<\phi^{\prime \prime}(t)<1 \forall t \in \mathbb{R} \text {. }
$$

Remark. From (4.4), the functions $\phi(t)$ and $\frac{t^{2}}{2}-\phi(t)$ are strictly convex (i.e., the hypothesis (H5), which is strengthened).

Thanks to (4.4), with the notations of the preceding section, $J(f)$ can be written, for $f \in H^{1}(\Omega)$, as

$$
J(f)=\inf _{b \in L^{2}(\Omega)^{2}} \int_{\Omega}(p-f)^{2}+\int_{\Omega}\left(\frac{|b-D f|^{2}}{2}+\psi(b)\right) d x d y .
$$

Proposition 4.1. For fixed $b$ in $L^{2}(\Omega)^{2}$ and for $p$ satisfying (4.2), the problem

$$
\inf _{f \in H^{1}(\Omega)} \int_{\Omega}\left((p-f)^{2}+\frac{|b-D f|^{2}}{2}\right) d x d y
$$


has a unique solution $f_{b} \in H^{1}(\Omega)$ verifying the Euler equation

$$
-\triangle f_{b}+2 f_{b}=2 p-\operatorname{div} b \quad \text { in } \mathcal{D}^{\prime}(\Omega) .
$$

Proof. The functional

$$
J_{b}(f)=\int_{\Omega}\left((p-f)^{2}+\frac{|b-D f|^{2}}{2}\right) d x d y
$$

being continuous, strictly convex, and coercive on $H^{1}(\Omega)$, then, by the classical theory of calculus of variations, there exists a unique $f_{b} \in H^{1}(\Omega)$ such that

$$
J_{b}\left(f_{b}\right) \leq J_{b}(f) \quad \forall f \in H^{1}(\Omega),
$$

which is equivalent to

$$
2 \int_{\Omega}\left(f_{b}-p\right) f d x d y+\int_{\Omega}\left(D f_{b}-b\right) \cdot D f d x d y=0 \quad \forall f \in H^{1}(\Omega) .
$$

Then we obtain (4.6), choosing $f \in \mathcal{D}(\Omega)$.

Remark. For the moment, we will not include in (4.6) the usual condition on the boundary $\partial \Omega$ of $\Omega$ : $\frac{\partial f}{\partial \eta}(x)=0$, the $H^{1}$-regularity of $f_{b}$ being insufficient to define the value on the boundary of the normal derivative.

Hence, we have for all $f \in H^{1}(\Omega)$ and fixed $b$

$$
\int_{\Omega}\left(f_{b}-p\right)^{2} d x d y+\int_{\Omega} \frac{\left|b-D f_{b}\right|^{2}}{2} d x d y \leq \int_{\Omega}(f-p)^{2} d x d y+\int_{\Omega} \frac{|b-D f|^{2}}{2} d x d y
$$

and by adding $\psi(b)$ on each side of (4.8) and taking the infimum in $b$, for all $f \in H^{1}(\Omega)$, we obtain

$$
\begin{array}{r}
\inf _{b \in L^{2}(\Omega)^{2}} \int_{\Omega}\left(\left(f_{b}-p\right)^{2}+\frac{\left|b-D f_{b}\right|^{2}}{2}+\psi(b)\right) d x d y \\
\leq \int_{\Omega}\left((p-R f)^{2}+\phi(|D f|)\right) d x d y=J(f) .
\end{array}
$$

We recall that

$$
T(b)=\int_{\Omega}\left(\left(f_{b}-p\right)^{2}+\frac{1}{2}\left|b-D f_{b}\right|^{2}+\psi(b)\right) d x d y .
$$

Now we must prove that the problem $\inf _{b \in L^{2}(\Omega)^{2}} T(b)$ has a solution $b_{0}$, which will involve the existence of a function $f_{0}$ solution of the initial problem

$$
J\left(f_{0}\right) \leq J(f) \quad \forall f \in \mathcal{V} .
$$

First, we will state some properties of the dual function $\psi$.

LEMMA 4.2. If $\phi$ verifies (4.3) and (4.4), then the function $\psi$ defined by (3.4) has the following properties:

(4.10) $\xi^{*} \rightarrow \psi\left(\xi^{*}\right)$ is strictly convex,

(4.11) there exist constants $a_{i}^{\prime}>0$ and $b_{i}^{\prime} \geq 0$ such that

$$
a_{1}^{\prime}\left|\xi^{*}\right|-b_{1}^{\prime} \leq \psi\left(\xi^{*}\right) \leq a_{2}^{\prime}\left|\xi^{*}\right|+b_{2}^{\prime} \quad \forall \xi^{*} \in \mathbb{R}^{2} .
$$


Proof. We recall the definition of $\psi\left(\xi^{*}\right)$. If $l(\xi)$ denotes the strictly convex function (from (4.4))

$$
l(\xi)=\frac{|\xi|^{2}}{2}-\phi(|\xi|)
$$

then $\psi\left(\xi^{*}\right)$ is defined by

$$
\psi\left(\xi^{*}\right)=l^{*}\left(\xi^{*}\right)-\frac{\left|\xi^{*}\right|^{2}}{2}
$$

$\left(l^{*}\right.$ denotes the Fenchel-Legendre transform of $l$ ).

We prove (4.11):

$$
\begin{aligned}
\psi\left(\xi^{*}\right) & =\sup _{\xi}\left(\xi^{*} \cdot \xi-l(\xi)\right)-\frac{\left|\xi^{*}\right|^{2}}{2} \\
& =\sup _{t \geq 0} \sup _{|\xi|=t}\left(\xi^{*} \cdot \xi-\frac{|\xi|^{2}}{2}+\phi(|\xi|)\right)-\frac{\left|\xi^{*}\right|^{2}}{2} \\
& =\sup _{t \geq 0}\left(t\left|\xi^{*}\right|-\frac{t^{2}}{2}+\phi(t)\right)-\frac{\left|\xi^{*}\right|^{2}}{2}
\end{aligned}
$$

and since $\phi$ is even,

$$
\psi\left(\xi^{*}\right)=\sup _{t \in \mathbb{R}}\left(t\left|\xi^{*}\right|-\frac{t^{2}}{2}+\phi(t)\right)-\frac{\left|\xi^{*}\right|^{2}}{2} .
$$

Hence, with (4.3), we have

$$
\begin{array}{r}
-b_{1}-\frac{\left|\xi^{*}\right|^{2}}{2}+\sup _{t \in \mathbb{R}}\left(a_{1}|t|+\left|\xi^{*}\right| t-\frac{t^{2}}{2}\right) \leq \psi\left(\xi^{*}\right) \\
\leq b_{2}-\frac{\left|\xi^{*}\right|^{2}}{2}+\sup _{t \in \mathbb{R}}\left(a_{2}|t|+\left|\xi^{*}\right| t-\frac{t^{2}}{2}\right) .
\end{array}
$$

The supremum in the right-hand side of (4.12) is achieved for $\bar{t}=a_{2}+\left|\xi^{*}\right|$, and its value is $\frac{1}{2}\left(a_{2}+\left|\xi^{*}\right|\right)^{2}$; hence, with (4.12),

$$
\psi\left(\xi^{*}\right) \leq b_{2}-\frac{\left|\xi^{*}\right|^{2}}{2}+\frac{1}{2}\left(a_{2}+\left|\xi^{*}\right|\right)^{2}=b_{2}+\frac{1}{2} a_{2}^{2}+a_{2}\left|\xi^{*}\right|,
$$

from which we obtain the second inequality of (4.11), with $a_{2}^{\prime}=a_{2}$ and $b_{2}^{\prime}=b_{2}+\frac{1}{2} a_{2}^{2}$. The first inequality of (4.11) can be proved in the same way. The proof of (4.10) follows from (4.4) and by a classical argument of convex analysis. From (4.4), we have that the function $\xi \rightarrow l(\xi)$ is strictly convex; hence, $l^{*}\left(\xi^{*}\right)$ is of class $C^{2}$ (by Rockfellar [21], Dacorogna [10]) and

$$
\begin{aligned}
\nabla \psi\left(\xi^{*}\right) & =\nabla l^{*}\left(\xi^{*}\right)-\xi^{*}, \\
\nabla^{2} \psi\left(\xi^{*}\right) & =\nabla^{2} l^{*}\left(\xi^{*}\right)-I .
\end{aligned}
$$

Moreover, since $l(\xi)$ is strictly convex, $\nabla l(\xi)$ is strictly monotonic and then, for each $\xi^{*} \in \mathbb{R}^{2}$, there is a unique $\xi_{0} \in \mathbb{R}^{2}$ such that $\xi^{*}=\nabla l\left(\xi_{0}\right)$, or equivalently, $\nabla l^{*}\left(\xi^{*}\right)=\xi_{0}$. Hence, we get

$$
\nabla \psi\left(\xi^{*}\right)=\xi_{0}-\nabla l\left(\xi_{0}\right)=-\nabla(\phi(|\xi|))_{\xi=\xi_{0}}=-\frac{\phi^{\prime}\left(\left|\xi_{0}\right|\right)}{\left|\xi_{0}\right|} \xi_{0}
$$


and (see Crouzeix [9] for computational details)

$$
\nabla^{2} \psi\left(\xi^{*}\right)=\left(\nabla^{2} l\left(\xi_{0}\right)\right)^{-1}-I
$$

which can be also written, from the definition of $l(\xi)$, as

$$
\nabla^{2} \psi\left(\xi^{*}\right)=\left(I-\nabla^{2}(\phi(|\xi|))_{\xi=\xi_{0}}\right)^{-1}-I .
$$

Thanks to (4.4), it is then clear that the matrix $\nabla^{2} \psi\left(\xi^{*}\right)$ is symmetric positive definite; consequently, $\psi$ is strictly convex.

Remark. In Lemma $4.2, \xi_{0}$ is, in fact, the unique point realizing the supremum $\sup _{\xi}\left(\xi \cdot \xi^{*}-l(\xi)\right)=l^{*}\left(\xi^{*}\right)$.

Provided with the properties of the function $\psi$, we can now return to the study of the problem (4.9):

$$
\inf _{b \in L^{2}(\Omega)^{2}}\left\{T(b)=\int_{\Omega}\left(\left(f_{b}-p\right)^{2}+\frac{\left|b-D f_{b}\right|^{2}}{2}+\psi(b)\right) d x d y\right\} .
$$

If $b_{n}$ is a minimizing sequence, then it is simple to deduce from (4.8) and (4.11) that $b_{n}$ and $f_{b_{n}}$ verify the estimates

$$
\begin{aligned}
& \left\|f_{b_{n}}\right\|_{L^{2}(\Omega)} \leq c, \\
& \left\|b_{n}\right\|_{L^{1}(\Omega)^{2}} \leq c,
\end{aligned}
$$

where $c$ is a constant which only depends on the data. But we cannot obtain an $H^{1}(\Omega)$-estimate for $f_{b_{n}}$ and an $L^{2}(\Omega)^{2}$-estimate for $b_{n}$, hence we must work on the nonreflexive space $L^{1}(\Omega)$ or on $\mathcal{M}_{b}(\Omega)$, the space of bounded measures. To overcome this difficulty, we regularize the problem by making a slight modification on the potential $\phi$. We introduce the function

$$
\phi_{\varepsilon}(t)=\phi(t)+\frac{\varepsilon}{2} t^{2}, \quad \varepsilon>0
$$

with which we associate

$$
\begin{aligned}
& l_{\varepsilon}(\xi)=\frac{|\xi|^{2}}{2}-\phi_{\varepsilon}(|\xi|), \\
& \psi_{\varepsilon}\left(\xi^{*}\right)=l_{\varepsilon}^{*}(\xi)-\frac{|\xi|^{2}}{2} .
\end{aligned}
$$

The function $\psi_{\varepsilon}$ has the same properties as $\psi$ if we modify and replace (4.4) by the following:

$(4.4)_{\varepsilon}$ There is $\varepsilon_{0}$, with $0<\varepsilon_{0}<1$ such that $0<\phi^{\prime \prime}(t)<1-\varepsilon_{0}$, for all $t \in \mathbb{R}$.

The assumption $(4.4)_{\varepsilon}$ is not restrictive, because we can always change the weighting parameter in the energy $J_{\alpha}(f)$, to have $(4.4)_{\varepsilon}$ verified.

By proceeding as in Lemma 4.2, it is easy to see, for $\varepsilon \leq \varepsilon_{0}$, that

$$
\begin{gathered}
\xi^{*} \rightarrow \psi_{\varepsilon}\left(\xi^{*}\right) \text { is strictly convex, } \\
-b_{1}+\frac{a_{1}^{2}}{2(1-\varepsilon)}+\frac{a_{1}}{1-\varepsilon}\left|\xi^{*}\right|+\frac{\varepsilon}{2(1-\varepsilon)}\left|\xi^{*}\right|^{2} \leq \psi_{\varepsilon}\left(\xi^{*}\right) \\
\leq b_{2}+\frac{a_{2}^{2}}{2(1-\varepsilon)}+\frac{a_{2}}{1-\varepsilon}\left|\xi^{*}\right|+\frac{\varepsilon}{2(1-\varepsilon)}\left|\xi^{*}\right|^{2},
\end{gathered}
$$


and the regularized problem associated with $T(b)$ is

$$
\inf _{b \in L^{2}(\Omega)^{2}}\left\{T_{\varepsilon}(b)=\int_{\Omega}\left(\left(f_{b}-p\right)^{2}+\frac{\left|b-D f_{b}\right|^{2}}{2}+\psi_{\varepsilon}(b)\right) d x d y\right\},
$$

for which we state the following proposition.

PROPOSITION 4.3. Under the assumptions (4.2), (4.3), and $(4.4)_{\varepsilon}$, the problem (4.15) has a unique solution $b_{\varepsilon}$ and there exists a constant $c$, independent of $\varepsilon$, such that

$$
\begin{aligned}
& \varepsilon\left\|b_{\varepsilon}\right\|_{L^{2}(\Omega)^{2}}^{2} \leq c, \\
& \varepsilon\left\|D f_{b_{\varepsilon}}\right\|_{L^{2}(\Omega)^{2}}^{2} \leq c, \\
& \left\|b_{\varepsilon}\right\|_{L^{1}(\Omega)^{2}} \leq c \\
& \left\|f_{b_{\varepsilon}}\right\|_{L^{2}(\Omega)} \leq c
\end{aligned}
$$

Proof. The functional $T_{\varepsilon}(b)$ is strictly convex since, from (4.6), the map $b \rightarrow f_{b}$ is affine from $L^{2}(\Omega)$ to $H^{1}(\Omega)$ and the function $\psi_{\varepsilon}$ is strictly convex (from $(4.10)_{\varepsilon}$ ).

Moreover, from $(4.11)_{\varepsilon}$, for each $b \in L^{2}(\Omega)^{2}$ there are some coefficients $a_{1}^{\prime}>0$ and $b_{1}^{\prime}$ such that

$$
\frac{\varepsilon}{2}\|b\|_{L^{2}(\Omega)^{2}}^{2}+a_{1}^{\prime}\|b\|_{L^{1}(\Omega)^{2}}-b_{1}^{\prime} \leq T_{\varepsilon}(b) ;
$$

hence, for fixed $\varepsilon$, the minimizing sequences $b_{\varepsilon}^{n}$ from (4.15) are bounded in $L^{2}(\Omega)^{2}$; therefore, there exist $b_{\varepsilon} \in L^{2}(\Omega)^{2}$ and a subsequence denoted also by $b_{\varepsilon}^{n}$ such that $b_{\varepsilon}^{n} \stackrel{w}{\longrightarrow} b_{\varepsilon}$ in $L^{2}(\Omega)$ weak. Since to the strict convexity of $T_{\varepsilon}, b_{\varepsilon}$ is unique, the entire sequence $b_{\varepsilon}^{n}$ converges to $b_{\varepsilon}$, and

$$
T_{\varepsilon}\left(b_{\varepsilon}\right) \leq \lim _{n \rightarrow \infty} T_{\varepsilon}\left(b_{\varepsilon}^{n}\right)=\inf _{b} T_{\varepsilon}(b) \leq T_{\varepsilon}(b) \quad \forall b \in L^{2}(\Omega)^{2} ;
$$

that is, $b_{\varepsilon}$ is the unique solution of (4.15):

$$
\begin{gathered}
\int_{\Omega}\left(\left(f_{b_{\varepsilon}}-p\right)^{2}+\frac{\left|b_{\varepsilon}-D f_{b_{\varepsilon}}\right|^{2}}{2}+\psi_{\varepsilon}\left(b_{\varepsilon}\right)+\frac{\varepsilon}{2}\left|b_{\varepsilon}\right|^{2}\right) d x d y \\
\leq \int_{\Omega}\left(\left(f_{b}-p\right)^{2}+\frac{\left|b-D f_{b}\right|^{2}}{2}+\psi_{\varepsilon}(b)+\frac{\varepsilon}{2}|b|^{2}\right) d x d y \quad \forall b \in L^{2}(\Omega)^{2} .
\end{gathered}
$$

Choosing, for example, $b=0$ in (4.17), it is clear that there is a constant $c$, independent of $\varepsilon$, such that (4.16) is verified.

The following theorem examines the optimality condition satisfied by $b_{\varepsilon}$.

THEOREM 4.4. The solution $b_{\varepsilon}$ of the problem (4.15) verifies the optimality condition

$$
(1+\varepsilon) b_{\varepsilon}+D \psi_{\varepsilon}\left(b_{\varepsilon}\right)-D f_{b_{\varepsilon}}=0 \quad \text { a.e. }(x, y) \in \Omega .
$$

Proof. To simplify the notations, we denote $f_{\varepsilon}=f_{b_{\varepsilon}}$; then let us consider a variation of $b_{\varepsilon}$ of the form $b_{\theta}=b_{\varepsilon}+\theta q$, where $\theta \in \mathbb{R}$ and $q \in L^{2}(\Omega)^{2}$. Denoting $f_{\theta}=f_{b_{\theta}}$, it is clear, thanks to the linearity of formula (4.6), that

$$
f_{\theta}=f_{\varepsilon}+\theta h
$$

where $h$ verifies

$$
-\triangle h+2 h=-\operatorname{div} q \text { in } H^{1}(\Omega)^{\prime} \quad\left(\text { the dual of } H^{1}(\Omega)\right) .
$$


With this remark

$$
\begin{gathered}
\frac{T_{\varepsilon}\left(b_{\theta}\right)-T_{\varepsilon}\left(b_{\varepsilon}\right)}{\theta} \\
(4.21)=\int_{\Omega}\left(2 f_{\varepsilon}-2 p+\theta h\right) h d x d y+\int_{\Omega}\left(2 b_{\varepsilon}-2 D f_{\varepsilon}+\theta(q-D h)\right) \cdot(q-D h) d x d y \\
+\frac{1}{\theta} \int_{\Omega}\left(\psi\left(b_{\varepsilon}+\theta q\right)-\psi\left(b_{\varepsilon}\right)\right) d x d y .
\end{gathered}
$$

Now, as $\theta \rightarrow 0$, the sum of the two first integrals converges to

$$
2 \int_{\Omega}\left(\left(f_{\varepsilon}-p\right) h+\left(b_{\varepsilon}-D f_{\varepsilon}\right) \cdot(q-D h)\right) d x d y .
$$

For the third integral, according to a result of convex analysis of Tahraoui [25], we have from $(4.10)_{\varepsilon}$ and $(4.11)_{\varepsilon}$ that there exist two constants $a(\varepsilon), b(\varepsilon) \geq 0$, such that

$$
\left|\nabla \psi_{\varepsilon}(b)\right| \leq a(\varepsilon)|b|+b(\varepsilon) \forall b \in \mathbb{R}^{2} .
$$

Hence, thanks to (4.16) and the Lebesgue dominated convergence theorem, we can pass to the limit in the third integral and obtain

$$
\lim _{\theta \rightarrow 0} \frac{1}{\theta}\left(T_{\varepsilon}\left(b_{\theta}\right)-T_{\varepsilon}\left(b_{\varepsilon}\right)\right)=2 \int_{\Omega}\left(\left(f_{\varepsilon}-p\right) h+\left(b_{\varepsilon}-D f_{\varepsilon}\right) \cdot(q-D h)+D \psi_{\varepsilon}\left(b_{\varepsilon}\right) q\right) d x d y
$$

But with the Euler equation of (4.7),

$$
2 \int_{\Omega}\left(f_{\varepsilon}-p\right) h d x d y=-\int_{\Omega}\left(D f_{\varepsilon}-b_{\varepsilon}\right) \cdot D h d x d y .
$$

Therefore, since $b_{\varepsilon}$ is a critical point of $T$, for all $q \in L^{2}(\Omega)^{2}$,

$$
\lim _{\theta \rightarrow 0} \frac{1}{\theta}\left(T_{\varepsilon}\left(b_{\theta}\right)-T_{\varepsilon}\left(b_{\varepsilon}\right)\right)=\int_{\Omega}\left(\left(b_{\varepsilon}-D f_{\varepsilon}\right)+D \psi_{\varepsilon}\left(b_{\varepsilon}\right)\right) q d x d y=0,
$$

which implies the relation we wanted to prove:

$$
b_{\varepsilon}-D f_{\varepsilon}+D \psi_{\varepsilon}\left(b_{\varepsilon}\right)=0 .
$$

In the following corollary, we express $D \psi_{\varepsilon}\left(b_{\varepsilon}\right)$ in terms of $D f_{\varepsilon}$ and $\phi^{\prime}\left(\left|D f_{\varepsilon}\right|\right)$. COROLLARY 4.5. The optimality condition (4.25) can be written as

$$
b_{\varepsilon}=\left((1-\varepsilon)-\frac{\phi^{\prime}\left(\left|D f_{\varepsilon}\right|\right)}{\left|D f_{\varepsilon}\right|}\right) D f_{\varepsilon} .
$$

Proof. We recall that

$$
\psi_{\varepsilon}\left(\xi^{*}\right)=l_{\varepsilon}^{*}\left(\xi^{*}\right)-\frac{\left|\xi^{*}\right|^{2}}{2}
$$

where

$$
l_{\varepsilon}(\xi)=\frac{|\xi|^{2}}{2}-\phi(|\xi|)-\frac{\varepsilon}{2}|\xi|^{2} .
$$

Thanks to $(4.3)$ and $(4.4)_{\varepsilon}$, the function $l_{\varepsilon}$ is strictly convex; hence, $l_{\varepsilon}^{*}$ and $\psi_{\varepsilon}$ are differentiable and

$$
D \psi_{\varepsilon}\left(\xi^{*}\right)=D l_{\varepsilon}^{*}\left(\xi^{*}\right)-\xi^{*} .
$$


But

$$
l_{\varepsilon}^{*}\left(\xi^{*}\right)=\sup _{\xi}\left(\xi^{*} \cdot \xi-l_{\varepsilon}(\xi)\right) .
$$

Then $D l_{\varepsilon}^{*}\left(\xi^{*}\right)=\xi_{\varepsilon}$, where $\xi_{\varepsilon}$ is the unique point realizing the $\sup _{\xi}\left(\xi^{*} \cdot \xi-l_{\varepsilon}(\xi)\right)$; that is, $\xi^{*}-D l_{\varepsilon}\left(\xi_{\varepsilon}\right)=0$ or

$$
\xi^{*}=(1-\varepsilon) \xi_{\varepsilon}-\xi_{\varepsilon} \frac{\phi^{\prime}\left(\left|\xi_{\varepsilon}\right|\right)}{\left|\xi_{\varepsilon}\right|}=0
$$

Denoting

$$
\mathcal{L}_{\varepsilon}(\xi)=(1-\varepsilon) \xi-\xi \frac{\phi^{\prime}(|\xi|)}{|\xi|}
$$

thanks to $(4.4)_{\varepsilon}, \mathcal{L}_{\varepsilon}$ is invertible and (4.28) is equivalent to

$$
\xi_{\varepsilon}=\mathcal{L}^{-1}\left(\xi^{*}\right) \text { and } D \psi_{\varepsilon}\left(\xi^{*}\right)=\mathcal{L}^{-1}\left(\xi^{*}\right)-\xi^{*} .
$$

With the optimality condition, we have the following sequence of equalities:

$$
\begin{array}{r}
D \psi_{\varepsilon}\left(b_{\varepsilon}\right)+b_{\varepsilon}-D f_{\varepsilon}=0, \\
\mathcal{L}^{-1}\left(b_{\varepsilon}\right)-b_{\varepsilon}+b_{\varepsilon}-D f_{\varepsilon}=0, \\
\mathcal{L}^{-1}\left(b_{\varepsilon}\right)=D f_{\varepsilon}, \\
b_{\varepsilon}=\mathcal{L}\left(D f_{\varepsilon}\right)=(1-\varepsilon) D f_{\varepsilon}-D f_{\varepsilon} \frac{\phi^{\prime}\left(\left|D f_{\varepsilon}\right|\right)}{\left|D f_{\varepsilon}\right|}, \\
b_{\varepsilon}=\left((1-\varepsilon)-\frac{\phi^{\prime}\left(\left|D f_{\varepsilon}\right|\right)}{\left|D f_{\varepsilon}\right|}\right) D f_{\varepsilon} .
\end{array}
$$

Now, to prove the existence of a solution for the initial problem (3.13), it remains to study the behavior of $f_{\varepsilon}$ and $b_{\varepsilon}$ when $\varepsilon \rightarrow 0$. The system linking $f_{\varepsilon}$ and $b_{\varepsilon}$ consists of two equations, namely, (4.6) and (4.26).

From (4.26) we have

$$
\operatorname{div} b_{\varepsilon}=(1-\varepsilon) \triangle f_{\varepsilon}-\operatorname{div}\left(\frac{\phi^{\prime}\left(\left|D f_{\varepsilon}\right|\right)}{\left|D f_{\varepsilon}\right|} D f_{\varepsilon}\right) .
$$

Putting this equality in (4.6), we get

$$
\varepsilon \triangle f_{\varepsilon}+\operatorname{div}\left(\frac{\phi^{\prime}\left(\left|D f_{\varepsilon}\right|\right)}{\left|D f_{\varepsilon}\right|} D f_{\varepsilon}\right)=2\left(f_{\varepsilon}-p\right) .
$$

Then, (4.29) is exactly the Euler equation associated with the problem

$$
\inf \left\{J_{\varepsilon}(f) ; f \in H^{1}(\Omega)\right\},
$$

where

$$
J_{\varepsilon}(f)=\int_{\Omega}(p-f)^{2} d x d y+\int_{\Omega} \phi(|D f|) d x d y+\frac{\varepsilon}{2} \int_{\Omega}|D f|^{2} d x d y
$$


Otherwise, we remark, thanks to (4.26), that

$$
\begin{aligned}
J_{\varepsilon}\left(f_{\varepsilon}\right) & =\int_{\Omega}\left(p-f_{\varepsilon}\right)^{2} d x d y+\int_{\Omega}\left(\phi\left(\left|D f_{\varepsilon}\right|\right)+\frac{\varepsilon}{2}\left|D f_{\varepsilon}\right|^{2}\right) d x d y \\
& =\int_{\Omega}\left(p-f_{\varepsilon}\right)^{2} d x d y+\inf _{b \in L^{2}(\Omega)^{2}} \int_{\Omega}\left(\frac{\left|b-D f_{\varepsilon}\right|^{2}}{2}+\psi_{\varepsilon}(b)\right) d x d y \\
& =\int_{\Omega}\left(\left(p-f_{\varepsilon}\right)^{2}+\frac{\left|b_{\varepsilon}-D f_{\varepsilon}\right|^{2}}{2}+\psi_{\varepsilon}\left(b_{\varepsilon}\right)\right) d x d y \\
& =\inf _{b \in L^{2}(\Omega)^{2}} T_{\varepsilon}(b) \leq J_{\varepsilon}(f) \quad \forall f \in H^{1}(\Omega) .
\end{aligned}
$$

Thanks to classical results of regularity, the solution $f_{\varepsilon}$ of (4.29) belongs to $C^{2}(\Omega)$ (see, for example, Ladyzenskaya and Uralceva [17] or Gilbarg and Trudinger [16]). Moreover, we can easily obtain an $L^{\infty}$-estimate for $f_{\varepsilon}$.

Proposition 4.6. If $p$ verifies (4.2), then the solution $f_{\varepsilon}$ of (4.29) satisfies

$$
0 \leq f_{\varepsilon}(x, y) \leq 1 \quad \text { a.e. }(x, y) \in \Omega .
$$

Proof. Let us show, for example, that $f_{\varepsilon}(x, y) \leq 1 \forall(x, y) \in \Omega$. The other inequality can be proved in the same way.

$f_{\varepsilon}$ is a solution of the variational problem

$2 \int_{\Omega}\left(f_{\varepsilon}-p\right) v d x d y+\int_{\Omega} \frac{\phi^{\prime}\left(\left|D f_{\varepsilon}\right|\right)}{\left|D f_{\varepsilon}\right|} D f_{\varepsilon} \cdot D v d x d y+\varepsilon \int_{\Omega} D f_{\varepsilon} \cdot D v d x d y=0 \forall v \in H^{1}(\Omega)$.

In (4.32), we choose $v=\left(f_{\varepsilon}-1\right)_{+} \geq 0$; according to Stamppachia [24], $v \in H^{1}(\Omega)$ and (4.32) can be written as

$$
\varepsilon \int_{f_{\varepsilon}>1}\left|D f_{\varepsilon}\right|^{2} d x d y+\int_{f_{\varepsilon}>1} \phi^{\prime}\left(\left|D f_{\varepsilon}\right|\right) d x d y=-2 \int_{f_{\varepsilon}>1}\left(f_{\varepsilon}-p\right)\left(f_{\varepsilon}-1\right)_{+} .
$$

But, by hypothesis, $\phi^{\prime}(t) \geq 0$ on $\mathbb{R}^{+}$(see (4.3)) and $0 \leq p(x, y) \leq 1$ a.e. $(x, y) \in \Omega$. Then $\left(f_{\varepsilon}-p\right)(x, y) \geq 0$ a.e. $(x, y) \in\left\{(x, y) ; f_{\varepsilon}>1\right\}$, which implies, from (4.33), that

$$
\int_{f_{\varepsilon}>1}\left|D f_{\varepsilon}\right|^{2} d x d y \leq 0
$$

from this, we have that $D f_{\varepsilon}(x, y)=0 \forall(x, y) \in\{(x, y) ; f(x, y)>1\}$; i.e., $\left(f_{\varepsilon}-1\right)_{+}=0$, which is equivalent to $f_{\varepsilon}(x, y) \leq 1$ a.e. $(x, y) \in \Omega$.

The following estimates are more delicate and are based on a very fine perturbation lemma due to Temam [12], [26]. This lemma is rather technical, and we will make a sketch of the proof in Appendix A.

PROPOSITION 4.7. If $p \in W^{1, \infty}$, then for every open set $\mathcal{O}$ relatively compact in $\Omega$, there is a constant $K=K\left(\mathcal{O}, \Omega,\|p\|_{W^{1, \infty}}\right)$ such that

$$
\begin{gathered}
\left\|f_{\varepsilon}\right\|_{W^{1, \infty}(\mathcal{O})} \leq K, \\
\left\|f_{\varepsilon}\right\|_{H^{2}(\mathcal{O})} \leq K .
\end{gathered}
$$

This proposition allows us to pass to the limit on $f_{\varepsilon}$ and $b_{\varepsilon}$ when $\varepsilon \rightarrow 0$. Besides the estimates (4.31), (4.34), and (4.35), we can add, thanks to (4.3):

$$
\left\|f_{\varepsilon}\right\|_{W^{1,1}(\Omega)} \leq c \quad(c \text { independent of } \varepsilon) .
$$


With these estimates, we can state, using the classical results of compactness and the diagonal process, that there is a function $f_{0}$ and a sequence $\varepsilon_{m} \rightarrow 0$ such that

$$
\begin{aligned}
& f_{\varepsilon_{m}} \rightarrow f_{0} \text { in } L^{\infty}(\Omega) \text { weak-star, } \\
& D f_{\varepsilon_{m}} \rightarrow D f_{0} \text { in } L^{\infty}(\mathcal{O}) \text { weak-star } \forall \mathcal{O} \subset \overline{\mathcal{O}} \subset \Omega, \\
& f_{\varepsilon_{m}} \rightarrow f_{0} \text { in } H^{2}(\mathcal{O}) \text { weak } \forall \mathcal{O} \subset \overline{\mathcal{O}} \subset \Omega, \\
& f_{\varepsilon_{m}} \rightarrow f_{0} \text { in } L^{1}(\Omega) \text { strong, } \\
& f_{\varepsilon_{m}}\left|\mathcal{O} \rightarrow f_{0}\right| \mathcal{O} \text { in } H^{1}(\mathcal{O}) \text { strong } \forall \mathcal{O} \subset \overline{\mathcal{O}} \subset \Omega, \\
& f_{\varepsilon_{m}}(x, y) \rightarrow f_{0}(x, y) \text { a.e. }(x, y), \\
& D f_{\varepsilon_{m}}(x, y) \rightarrow D f_{0}(x, y) \text { a.e. }(x, y),
\end{aligned}
$$

and we have the following result.

THEOREM 4.8. Under the previous assumptions, (4.2), (4.3), and (4.4), and if $p \in W^{1, \infty}(\Omega)$, then the function $f_{0}$ defined before belongs to $W^{1,1}(\Omega) \cap L^{\infty}(\Omega)$ and is the unique solution of the initial optimization problem

$$
\inf \left\{J(f)=\int_{\Omega}(p-f)^{2} d x d y+\int_{\Omega} \phi(|D f|) d x d y, f \in L^{2}(\Omega), D f \in L^{1}(\Omega)^{2}\right\} .
$$

Proof. By the Fatou lemma, (4.36) and (4.43), it is clear that $f_{0}$ belongs to $W^{1,1}(\Omega) \cap L^{\infty}(\Omega)$ (we have, moreover, that $\left.f_{0}\right|_{\mathcal{O}} \in H^{2}(\mathcal{O}) \cap W^{1, \infty}(\mathcal{O})$, for all $\mathcal{O}$ with $\mathcal{O} \subset \overline{\mathcal{O}} \subset \Omega)$. $f_{0}$ is a solution of (4.44). In fact, $f_{\varepsilon_{m}}$ is the solution of the variational problem (4.32). Thanks to the Tahraoui result mentioned before, the assumptions (4.3) and (4.4) imply that there exists a constant $M>0$ such that $\left|\phi^{\prime}(t)\right| \leq M$, for all $t \in \mathbb{R}$. Therefore, with the convergences (4.37)-(4.43) and the Lebesgue dominated convergence theorem, we can pass to the limit in (4.32) and obtain

$$
2 \int_{\Omega}\left(f_{0}-p\right)^{2} v d x d y+\int_{\Omega} \frac{\phi^{\prime}\left(\left|D f_{0}\right|\right)}{\left|D f_{0}\right|} D f_{0} \cdot D v d x d y=0 \quad \forall v \in H^{1}(\Omega) .
$$

By density, (4.45) is true for all $v \in L^{2}(\Omega)$ with $D v \in L^{1}(\Omega)^{2}$, and since the problem is strictly convex, $f_{0}$ is the unique solution of (4.44); moreover, $0 \leq f(x, y) \leq 1$ a.e. $(x, y) \in \Omega$.

The previous results imply some convergence properties for the sequence of the dual variables $b_{\varepsilon}$. In fact, we have proved that $b_{\varepsilon}$ verifies

$$
b_{\varepsilon}=\left((1-\varepsilon)-\frac{\phi^{\prime}\left(\left|D f_{\varepsilon}\right|\right)}{\left|D f_{\varepsilon}\right|}\right) D f_{\varepsilon}
$$

and

$$
\begin{gathered}
J_{\varepsilon}\left(f_{\varepsilon}\right)=J\left(f_{\varepsilon}\right)+\frac{\varepsilon}{2} \int_{\Omega}\left|D f_{\varepsilon}\right|^{2} d x d y=\int_{\Omega}\left(p-f_{\varepsilon}\right)^{2} d x d y \\
+\inf _{b \in L^{2}(\Omega)^{2}} \int_{\Omega}\left(\frac{\left|b-D f_{\varepsilon}\right|^{2}}{2}+\psi_{\varepsilon}(b)\right) d x d y=\inf _{b \in L^{2}(\Omega)^{2}} T_{\varepsilon}(b) \leq J_{\varepsilon}(f) \forall f \in H^{1}(\Omega) .
\end{gathered}
$$

If $\varepsilon \rightarrow 0$, we deduce from (4.46) that $b_{\varepsilon}(x, y) \rightarrow b_{0}(x, y)$ a.e. $(x, y) \in \Omega$, where

$$
b_{0}(x, y)=\left(1-\frac{\phi^{\prime}\left(\left|D f_{0}(x, y)\right|\right)}{\left|D f_{0}(x, y)\right|}\right) D f_{0}(x, y) .
$$


The sequence of equalities in (4.47) proves that $f_{\varepsilon}$ is a minimizing sequence for the problem $\inf _{f} J(f)$, and that

$$
\begin{aligned}
\int_{\Omega} \phi\left(\left|D f_{0}\right|\right) d x d y & =\lim _{\varepsilon \rightarrow 0} \inf _{b \in L^{2}(\Omega)^{2}} \int_{\Omega}\left(\frac{\left|b-D f_{0}\right|^{2}}{2}+\psi_{\varepsilon}(b)\right) d x d y \\
& =\lim _{\varepsilon \rightarrow 0} \int_{\Omega}\left(\phi\left(\left|D f_{\varepsilon}\right|\right)+\frac{\varepsilon}{2}\left|D f_{\varepsilon}\right|^{2}\right) d x d y .
\end{aligned}
$$

We will present more precisely some convergence results for $b_{\varepsilon}$ in the next section.

Remark. In Theorem 4.8, we have obtained the existence under the condition $p \in W^{1, \infty}(\Omega)$. This is a restrictive condition, the most natural being $p \in L^{\infty}$. This restriction is due to the method; we can relax it by working on $B V(\Omega)$, the space of functions with bounded variation, and by using the notion of convex function of a measure [11], [18]. Or, by another point of view, we can solve the problem in the context of viscosity solutions (see [8] for the general theory and [18] for applications to image analysis). Nevertheless, with the assumption $p \in W^{1, \infty}(\Omega)$, we have obtained the regularity result $f_{0} \in H^{2}(\mathcal{O}) \cap W^{1, \infty}(\mathcal{O})$ for all $\mathcal{O} \subset \overline{\mathcal{O}} \subset \Omega$; that is, the process is regularizing.

5. Description and convergence of the algorithm. In this section, we are working in the context of Theorem 4.8, and we assume the existence and uniqueness of a function $f_{0} \in W^{1,1}(\Omega) \cap L^{\infty}(\Omega)$, which is the solution of

$$
\inf \left\{J(f)=\int_{\Omega}(p-f)^{2} d x d y+\int_{\Omega} \phi(|D f|) d x d y ; f \in L^{2}(\Omega), D f \in L^{1}(\Omega)^{2}\right\} .
$$

Using the previous results, we describe the algorithm for computing $f_{0}$. We denote $T(b, f)$, the functional defined on $L^{2}(\Omega)^{2} \times H^{1}(\Omega)$, by

$$
T(b, f)=\int_{\Omega}(p-f)^{2} d x d y+\frac{1}{2} \int_{\Omega}|D f-b|^{2} d x d y+\int_{\Omega} \psi(b) d x d y
$$

(with $\psi$ defined as before).

The iterative algorithm is as follows.

(i) $f^{0} \in H^{1}(\Omega)$ is arbitrarily given, with $0 \leq f^{0} \leq 1$.

(ii) $f^{n} \in H^{1}(\Omega)$ being calculated, we compute $b^{n+1}$ by solving the minimization problem

$$
T\left(b^{n+1}, f^{n}\right) \leq T\left(b, f^{n}\right) \forall b \in L^{2}(\Omega)^{2} .
$$

Equation (5.3), which is a strictly convex problem, has a unique solution $b^{n+1}$ satisfying the equation $b^{n+1}=D f^{n+1}-D \psi\left(b^{n+1}\right)$, or, by Corollary 4.5,

$$
b^{n+1}=\left(1-\frac{\phi^{\prime}\left(\left|D f^{n}\right|\right)}{\left|D f^{n}\right|}\right) D f^{n} .
$$

(iii) $f^{n+1}$ is therefore calculated as the solution of the problem

$$
T\left(b^{n+1}, f^{n+1}\right) \leq T\left(b^{n+1}, f\right) \forall f \in H^{1}(\Omega),
$$

which is equivalent to solving the variational problem

$$
\int_{\Omega}\left(D f^{n+1}-b^{n+1}\right) \cdot D f d x d y+2 \int_{\Omega}\left(f^{n+1}-p\right) f d x d y=0 \forall f \in H^{1}(\Omega) .
$$

Equation (5.6) has a unique solution $f^{n+1}$.

We denote by $U_{n}$ the sequence $U_{n}=T\left(b^{n+1}, f^{n}\right)$. 
LEMMA 5.1. The sequence $U_{n}$ is convergent.

Proof. We prove that $U_{n}$ is decreasing and bounded below. We have

$$
\begin{gathered}
U_{n-1}-U_{n}=T\left(b^{n}, f^{n-1}\right)-T\left(b^{n+1}, f^{n}\right), \\
U_{n-1}-U_{n}=\left(T\left(b^{n}, f^{n}\right)-T\left(b^{n+1}, f^{n}\right)\right)+\left(T\left(b^{n}, f^{n-1}\right)-T\left(b^{n}, f^{n}\right)\right) ;
\end{gathered}
$$

thanks to the definition of $b^{n+1}$ and $f^{n}$, we have for all $n>0$,

$$
\begin{aligned}
& A_{n}=T\left(b^{n}, f^{n}\right)-T\left(b^{n+1}, f^{n}\right) \geq 0, \\
& B_{n}=T\left(b^{n}, f^{n-1}\right)-T\left(b^{n}, f^{n}\right) \geq 0 .
\end{aligned}
$$

Therefore, $U_{n-1}-U_{n}=A_{n}+B_{n} \geq 0$; that is, $U_{n}$ is decreasing, and since

$$
\inf _{b} \int_{\Omega} \psi(b) d x d y>-\infty
$$

the sequence $U_{n}$ is bounded below and then is convergent.

LEMMA 5.2. The previous sequence $b^{n}$ verifies

$$
\lim _{n \rightarrow \infty}\left\|b^{n}-b^{n+1}\right\|_{L^{2}(\Omega)^{2}}=0 .
$$

Proof. We study the term $A_{n}$, which can be written as

$$
\begin{aligned}
A_{n} & =\int_{\Omega}\left(f^{n}-p\right)^{2} d x d y+\frac{1}{2} \int_{\Omega}\left|D f^{n}-b^{n}\right|^{2} d x d y+\int_{\Omega} \psi\left(b^{n}\right) d x d y \\
& -\int_{\Omega}\left(f^{n}-p\right)^{2} d x d y-\frac{1}{2} \int_{\Omega}\left|D f^{n}-b^{n+1}\right|^{2} d x d y-\int_{\Omega} \psi\left(b^{n+1}\right) d x d y, \\
A_{n} & =\int_{\Omega}\left(\frac{1}{2}\left|D f^{n}-b^{n}\right|^{2}+\psi\left(b^{n}\right)\right) d x d y-\int_{\Omega}\left(\frac{1}{2}\left|D f^{n}-b^{n+1}\right|^{2}+\psi\left(b^{n+1}\right)\right) d x d y .
\end{aligned}
$$

Denoting $h_{n}(b)=\frac{1}{2}\left|D f^{n}-b\right|^{2}+\psi(b)$, then

$$
A_{n}=\int_{\Omega}\left(h_{n}\left(b^{n}\right)-h_{n}\left(b^{n+1}\right)\right) d x d y .
$$

Thanks to the Taylor formula, there exists $c_{n}$ between $b^{n}$ and $b^{n+1}$ such that

$$
A_{n}=\int_{\Omega}\left(b^{n}-b^{n+1}\right) \cdot D h_{n}\left(b^{n+1}\right) d x d y+\frac{1}{2} \int_{\Omega}^{t}\left(b^{n}-b^{n+1}\right) \cdot D^{2} h_{n}\left(c_{n}\right)\left(b^{n}-b^{n+1}\right) d x d y .
$$

But $D h_{n}\left(b^{n+1}\right)=b^{n+1}-D f^{n}+D \psi\left(b^{n+1}\right)=0$, by the definition of $b^{n+1}$. Moreover, $D^{2} h_{n}(b)=I+D^{2} \psi(b) \geq I$, because $\psi$ is convex (by Lemma 4.2 ). Consequently,

$$
A_{n} \geq \int_{\Omega}\left|b^{n}-b^{n+1}\right|^{2} d x d y .
$$

Otherwise, $U_{n-1}-U_{n}=A_{n}+B_{n} \geq A_{n} \geq 0$, and since the sequence $U_{n}$ is convergent, $\lim _{n \rightarrow \infty} A_{n}=0$, which implies that

$$
\lim _{n \rightarrow \infty} \int_{\Omega}\left|b^{n}-b^{n+1}\right|^{2} d x d y=0 .
$$

In general, we cannot obtain a more precisely convergent theorem (for example, the convergence in $H^{1}(\Omega)$ ), without supposing more regularity on the solution. 
LEMma 5.3. If $0 \leq\left(\phi^{\prime}(t) / t\right) \leq 1 \forall t \geq 0$, then the sequence $f^{n}$ is bounded in $H^{1}(\Omega)$.

Proof. The proof is based on a recurrence process. In (5.6) we choose $f=f^{n+1}$; we get

$$
\int_{\Omega}\left(\left|D f^{n+1}\right|^{2}+2\left(f^{n+1}\right)^{2}\right) d x d y=\int_{\Omega}\left(b^{n+1} \cdot D f^{n+1}+2 p f^{n+1}\right) d x d y .
$$

With (5.4), and since $0 \leq\left(\phi^{\prime}(t) / t\right) \leq 1$ (in fact, in the applications, $\left(\phi^{\prime}(t) / t\right)$ decreases from 1 to 0 for $t \in] 0, \infty\left[\right.$ ), we have $\left|b^{n+1}\right| \leq\left|D f^{n}\right|$, which implies, with (5.8), that

$$
\int_{\Omega}\left(\left|D f^{n+1}\right|^{2}+2\left(f^{n+1}\right)^{2}\right) d x d y \leq \int_{\Omega}\left(\left|D f^{n}\right| \cdot\left|D f^{n+1}\right|+2|p|\left|f^{n+1}\right|\right) d x d y .
$$

Denoting $M=\max \left(2\|p\|_{L^{2}},\left\|f^{0}\right\|_{H^{1}}\right)$, we have

$$
\left\|f^{n}\right\|_{H^{1}(\Omega)} \leq M \forall n .
$$

In fact, (5.10) is true for $n=0$; suppose that (5.10) is true for $n$, and with (5.9),

$$
\begin{aligned}
\left\|f^{n+1}\right\|_{H^{1}}^{2} & \leq \int_{\Omega}\left(\left|D f^{n+1}\right|^{2}+2\left(f^{n+1}\right)^{2}\right) d x d y \\
& \leq M\left\|D f^{n+1}\right\|_{L^{2}}+2\|p\|_{L^{2}}\left\|f^{n+1}\right\|_{L^{2}} \leq M\left\|f^{n+1}\right\|_{H^{1}}
\end{aligned}
$$

from which $\left\|f^{n+1}\right\|_{H^{1}} \leq M$. Then (5.10) is true for all $n$.

Like a corollary of Lemma 5.3, we easily deduce that the sequence $b^{n}$ is bounded in $L^{2}(\Omega)^{2}$. The following theorem examines the convergence of $f^{n}$ to $f_{0}$, the solution of the problem (5.1); it is therefore necessary to add a slight regularity assumption on $f_{0}$.

THEOREM 5.4. If the solution $f_{0}$ of (5.1) belongs to $H^{1}(\Omega)$, then

i) $f^{n} \rightarrow f_{0}$ in $L^{2}(\Omega)$ strong;

ii) $D f^{n} \rightarrow D f_{0}$ in $L^{2}(\Omega)^{2}$ weak;

iii) $\lim _{n \rightarrow \infty} \int_{\Omega} \phi\left(\left|D f^{n}\right|\right) d x d y=\int_{\Omega} \phi\left(\left|D f_{0}\right|\right) d x d y$;

iv) $D f^{n} \rightarrow D f_{0}$ in $L^{1}(\Omega)^{2}$ strong.

Proof. We know that $f_{0}$ is the unique solution, belonging to

$$
\mathcal{V}=\left\{f \in L^{2}(\Omega), D f \in L^{1}(\Omega)^{2}\right\},
$$

of the variational problem

$$
\int_{\Omega} \frac{\phi^{\prime}\left(\left|D f_{0}\right|\right)}{\left|D f_{0}\right|} D f_{0} \cdot D f d x d y+2 \int_{\Omega}\left(f_{0}-p\right) f d x d y=0 \forall f \in H^{1}(\Omega) .
$$

With $b_{0}$ defined in the previous section, (5.11) is equivalent to (all the integrals have sense, because $f_{0} \in H^{1}(\Omega)$ )

$$
\int_{\Omega}\left(D f_{0} \cdot D f-b_{0} \cdot D f\right) d x d y+2 \int_{\Omega}\left(f_{0}-p\right) f d x d y=0 \forall f \in H^{1}(\Omega) .
$$


Otherwise, with (5.6), $f^{n}$ is defined by

$$
\int_{\Omega}\left(D f^{n} \cdot D f-b^{n} \cdot D f\right) d x d y+2 \int_{\Omega}\left(f^{n}-p\right) f d x d y=0 \forall f \in H^{1}(\Omega) .
$$

By subtracting (5.13) from (5.12), and choosing $f=f_{0}-f^{n}$, we get

$$
\int_{\Omega}\left|D f_{0}-D f^{n}\right|^{2} d x d y+2 \int_{\Omega}\left|f_{0}-f^{n}\right|^{2} d x d y-\int_{\Omega}\left(b^{n}-b_{0}\right) \cdot\left(D f^{n}-D f_{0}\right) d x d y=0 .
$$

With the definition of $b_{0}$ and $b^{n}$, by adding and subtracting $b^{n+1}$, it is easy to see that

$$
\begin{aligned}
\int_{\Omega}\left(b^{n}-b_{0}\right) \cdot\left(D f^{n}\right. & \left.-D f_{0}\right) d x d y \\
& =\int_{\Omega}\left|D f^{n}-D f_{0}\right|^{2} d x d y+\int_{\Omega}\left(b^{n}-b^{n+1}\right)\left(D f^{n}-D f_{0}\right) d x d y \\
& +\int_{\Omega}\left(\frac{\phi^{\prime}\left(\left|D f_{0}\right|\right)}{\left|D f_{0}\right|} D f_{0}-\frac{\phi^{\prime}\left(\left|D f^{n}\right|\right)}{\left|D f^{n}\right|} D f^{n}\right)\left(D f^{n}-D f_{0}\right) d x d y
\end{aligned}
$$

If we denote

$$
j(f)=\int_{\Omega} \phi(|D f|) d x d y
$$

then (5.14) can be written as

$$
\begin{aligned}
2 \int_{\Omega}\left(f^{n}-f_{0}\right)^{2} d x d y & +\int_{\Omega}\left(b^{n+1}-b^{n}\right)\left(D f^{n}-D f_{0}\right) d x d y \\
& +\left\langle j^{\prime}\left(f_{0}\right)-j^{\prime}\left(f^{n}\right), f^{n}-f_{0}\right\rangle=0 .
\end{aligned}
$$

Since $j$ is convex, the third integral in (5.15) is nonnegative and then

$$
2 \int_{\Omega}\left(f^{n}-f_{0}\right)^{2} d x d y+\int_{\Omega}\left(b^{n+1}-b^{n}\right) \cdot\left(D f^{n}-D f_{0}\right) d x d y \leq 0 .
$$

With Lemma 5.2, $\left(b^{n+1}-b^{n}\right) \stackrel{n \rightarrow \infty}{\rightarrow} 0$ in $L^{2}(\Omega)^{2}$ strong, and with Lemma 5.3 and the assumption $f_{0} \in H^{1}(\Omega)$, we have that $D f^{n}-D f_{0}$ is bounded in $L^{2}(\Omega)^{2}$; hence, by passing to the limit in (5.16), we get

$$
\lim _{n \rightarrow \infty} \int_{\Omega}\left(f^{n}-f_{0}\right)^{2} d x d y=0 .
$$

To prove ii), we remark, thanks to Lemma 5.3, that there is an $\tilde{f} \in H^{1}(\Omega)$ such that $f^{n} \rightarrow \tilde{f}$ (or for a subsequence) in $H^{1}(\Omega)$ weak and, with (5.17), that necessarily $\tilde{f}=f_{0}$, and that the entire sequence converges.

To prove iii), we deduce from (5.15) and (5.17) that

$$
\lim _{n \rightarrow \infty}\left\langle j^{\prime}\left(f_{0}\right)-j^{\prime}\left(f^{n}\right), f^{n}-f_{0}\right\rangle=0
$$

that is,

$$
\lim _{n \rightarrow \infty} \int_{\Omega}\left(\frac{\phi^{\prime}\left(D f_{0} \mid\right)}{\left|D f_{0}\right|} D f_{0}-\frac{\phi^{\prime}\left(\left|D f^{n}\right|\right)}{\left|D f^{n}\right|} D f^{n}\right) \cdot\left(D f^{n}-D f_{0}\right) d x d y=0
$$


and since $D f^{n} \rightarrow D f_{0}$ in $L^{2}(\Omega)^{2}$ weak, (5.18) implies that

$$
\lim _{n \rightarrow \infty} \int_{\Omega} \frac{\phi^{\prime}\left(\left|D f^{n}\right|\right)}{\left|D f^{n}\right|} D f^{n} \cdot\left(D f^{n}-D f_{0}\right) d x d y=0 .
$$

But, since $\phi$ is convex, we have

$$
\int_{\Omega}\left(\phi\left(\left|D f_{0}\right|\right)-\phi\left(\left|D f^{n}\right|\right)\right) d x d y \geq \int_{\Omega} \frac{\phi^{\prime}\left(\left|D f^{n}\right|\right)}{\left|D f^{n}\right|} D f^{n} \cdot\left(D f_{0}-D f^{n}\right) d x d y
$$

from which, with (5.19):

$$
\int_{\Omega} \phi\left(\left|D f_{0}\right|\right) d x d y \geq \varlimsup_{n \rightarrow \infty} \int_{\Omega} \phi\left(\left|D f^{n}\right|\right) d x d y .
$$

And, since we always have (thanks to the convexity of $\phi$ )

$$
\underline{\lim }_{n \rightarrow \infty} \int_{\Omega} \phi\left(\left|D f^{n}\right|\right) d x d y \geq \int_{\Omega} \phi\left(\left|D f_{0}\right|\right) d x d y,
$$

we get

$$
\lim _{n \rightarrow \infty} \int_{\Omega} \phi\left(\left|D f^{n}\right|\right) d x d y=\int_{\Omega} \phi\left(\left|D f_{0}\right|\right) d x d y .
$$

The proof of iv) is a consequence of the following result due to Visintin.

THEOREM 5.5 (Visintin [28, Thm. 3]). Let $\Phi$ be a strictly convex function from $\mathbb{R}^{2} \rightarrow \mathbb{R}$ and let $u_{n}$ be a sequence from $L^{1}(\Omega)^{2}$ such that

$$
\begin{aligned}
u_{n} \rightarrow u & \text { in } L^{1}(\Omega)^{2} \text { weak }, \\
\int_{\Omega} \Phi\left(u_{n}\right) d x d y & \rightarrow \int_{\Omega} \Phi(u) d x d y .
\end{aligned}
$$

Then $u_{n} \rightarrow u$ in $L^{1}(\Omega)$ strong.

To prove part iv), we apply the Visintin result with $u_{n}=D f^{n}$ and $\Phi(u)=$ $\phi(|D u|)$.

Remarks.

(1) $f_{0}$, the solution of the initial reconstruction problem (5.1), necessarily verifies, in the sense of distribution,

$$
2\left(p-f_{0}\right)-\operatorname{div}\left(\frac{\phi^{\prime}\left(D f_{0} \mid\right)}{\left|D f_{0}\right|} D f_{0}\right)=0 \text { in } \mathcal{D}^{\prime}(\Omega) .
$$

Since $p, f_{0} \in L^{\infty}(\Omega)$, we deduce from (5.21) that

$$
\operatorname{div}\left(\frac{\phi^{\prime}\left(D f_{0} \mid\right)}{\left|D f_{0}\right|} D f_{0}\right) \in L^{\infty}(\Omega)
$$

and then if $f_{0} \in H^{1}(\Omega)$, with a result of Lions and Magenes [19], we can give sense, on the boundary $\partial \Omega$ of $\Omega$, to the conormal derivative $\phi^{\prime}\left(\left|D f_{0}\right|\right) /\left|D f_{0}\right| D f_{0} \cdot n$ ( $n$ is the exterior normal to $\partial \Omega$ ). Multiplying (5.21) by $f \in H^{1}(\Omega)$ and integrating by parts, we get, with (5.12),

$$
\frac{\phi^{\prime}\left(\left|D f_{0}\right|\right)}{\left|D f_{0}\right|} \frac{\partial f_{0}}{\partial n}=0 \text { on } \partial \Omega
$$


and if $\lim _{t \rightarrow \infty} \phi^{\prime}(t) / t=0$ (by (2.12)), then with (5.22), we have either

$$
\frac{\partial f_{0}}{\partial n}=0 \text { on } \partial \Omega
$$

or

$$
\left|D f_{0}\right|=+\infty \text { on } \partial \Omega \text {. }
$$

Supposing that in a neighborhood of its boundary, the image does not present an edge, we can incorporate (5.23) like a boundary condition in the algorithm.

(2) If $\lim _{t \rightarrow 0} \phi^{\prime}(t) / t=1$ by $(2.7), \lim _{t \rightarrow \infty} \phi^{\prime}(t) / t=0$ by (2.12); then the function $\frac{\left|b_{0}\right|}{\left|D f_{0}\right|}(x, y)$ is an edge indicator which takes, roughly speaking, only the values 1 or 0 . In fact,

$$
\frac{\left|b_{0}\right|}{\left|D f_{0}\right|}(x, y)=\left(1-\frac{\phi^{\prime}\left(\left|D f_{0}\right|\right)}{\left|D f_{0}\right|}\right)(x, y)
$$

In a neighborhood of a pixel $(x, y)$ belonging to an edge, $\left|D f_{0}\right|$ is big and $\frac{\left|b_{0}\right|}{\left|D f_{0}\right|} \sim 1$, whereas in the interior of a homogeneous region, $\left|D f_{0}\right|(x, y)$ is small and $\frac{\left|b_{0}\right|}{\left|D f_{0}\right|}(x, y) \sim$ 0 .

(3) There are other dualities for introducing an auxiliary variable. For example, if $t \rightarrow \phi(\sqrt{t})$ is strictly concave, we can prove that there is a function $\psi$ strictly convex and decreasing such that

$$
\phi(t)=\inf _{b}\left(b t^{2}+\psi(b)\right) .
$$

This duality was exploited by Geman and Reynolds [15] and Charbonnier et al. [6].

6. The numerical approximation of the model. In this section we will present the numerical approximation, by using the finite difference method, for the Euler equation associated with the minimization reconstruction problem; that is,

$$
\lambda\left(R^{*} R f-R^{*} p\right)-\operatorname{div}\left(\frac{\phi^{\prime}(|D f|)}{|D f|} D f\right)=0,
$$

where the function $\phi$ is of the type of potential introduced in the previous sections (this equation is equivalent to (2.5), by taking $\lambda=\frac{2}{\alpha}$ ). For the tests, we have used $\phi_{1}(t)=\sqrt{1+t^{2}}$ in the convex case and $\phi_{2}(t)=\frac{t^{2}}{1+t^{2}}$, which is not convex.

Before starting with the algorithm, we recall some standard notation. Let

$$
\begin{aligned}
& \left.1^{0}\right) x_{i}=i h, y_{j}=j h, i, j=1,2, \ldots, N, \text { with } h>0 ; \\
& \left.2^{0}\right) f_{i j} \approx f\left(x_{i}, y_{j}\right), f_{i j}^{n} \approx f^{n}\left(x_{i}, y_{j}\right) ; \\
& \left.3^{0}\right) p_{i j} \approx p\left(x_{i}, y_{j}\right) ; \\
& \left.4^{0}\right) m(a, b)=\operatorname{minmod}(a, b)=\frac{\operatorname{sgn} a+\operatorname{sgn} b}{2} \min (|a|,|b|) ; \\
& \left.5^{0}\right) \triangle_{\mp}^{x} f_{i j}=\mp\left(f_{i \mp 1, j}-f_{i j}\right) \text { and } \triangle_{\mp}^{y} f_{i j}=\mp\left(f_{i, j \mp 1}-f_{i j}\right) .
\end{aligned}
$$
by

For the moment, we begin with the case $R=I$ and let $\psi$, the function, be defined

$$
\psi: \mathbb{R} \rightarrow \mathbb{R}, \quad \psi(t)= \begin{cases}\frac{\phi^{\prime}(t)}{t} & \text { if } t \neq 0 \\ \lim _{t \rightarrow 0} \frac{\phi^{\prime}(t)}{t} & \text { if } t=0\end{cases}
$$


Then, for each type of potential, $\phi_{1}$ and $\phi_{2}$, the function $\psi$ is positive and bounded on $\mathbb{R}$.

The numerical method is as follows. (We essentially adopt the method of Rudin, Osher, and Fatemi [23] to approximate the divergence term, and we use an iteration algorithm.)

We suppose that $\Omega$ is a rectangle. So, $\left(p_{i j}\right)_{i, j=1, N}$ is the initial discrete image such that $m_{1} \leq p_{i j} \leq m_{2}$, where $m_{2} \geq m_{1} \geq 0$. We will approach the numerical solution $\left(f_{i j}\right)_{i, j=1, N}$ by a sequence $\left(f_{i j}^{n}\right)_{i, j=1, N}$ for $n \rightarrow \infty$, which is obtained as follows.

1) $f^{0}$ is arbitrarily given, such that $m_{1} \leq f_{i j}^{0} \leq m_{2}$.

2) If $f^{n}$ is calculated, then we compute $f^{n+1}$ as a solution of the linear discrete problem

$$
\begin{aligned}
\lambda f_{i j}^{n+1} & -\frac{1}{h}\left\{\triangle_{-}^{x}\left[\psi\left(\left(\left(\frac{\triangle_{+}^{x} f_{i j}^{n}}{h}\right)^{2}+\left(m\left(\frac{\triangle_{+}^{y} f_{i j}^{n}}{h}, \frac{\triangle_{-}^{y} f_{i j}^{n}}{h}\right)\right)^{2}\right)^{\frac{1}{2}}\right)\left(\frac{\triangle_{+}^{x} f_{i j}^{n+1}}{h}\right)\right]\right\} \\
& -\frac{1}{h}\left\{\triangle_{-}^{y}\left[\psi\left(\left(\left(\frac{\triangle_{+}^{y} f_{i j}^{n}}{h}\right)^{2}+\left(m\left(\frac{\triangle_{+}^{x} f_{i j}^{n}}{h}, \frac{\triangle_{-}^{x} f_{i j}^{n}}{h}\right)\right)^{2}\right)^{\frac{1}{2}}\right)\left(\frac{\triangle_{+}^{y} f_{i j}^{n+1}}{h}\right)\right]\right\} \\
& =\lambda p_{i j},
\end{aligned}
$$

for $i, j=1, \ldots, N$ and with the boundary conditions obtained by reflection as

$$
f_{0 j}^{n}=f_{2 j}^{n}, f_{N+1, j}^{n}=f_{N-1, j}^{n}, f_{i 0}^{n}=f_{i 2}^{n}, f_{i N+1}^{n}=f_{i, N-1}^{n} .
$$

Remark. The algorithm described in the previous section allows us to compute $f^{n+1}$ by the formula (instead of (6.1))

$$
\lambda f_{i j}^{n+1}-\triangle f_{i j}^{n+1}=p_{i j}-\operatorname{div}\left[\left(1-\frac{\phi^{\prime}\left(\left|D f_{i j}^{n}\right|\right)}{\left|D f_{i j}^{n}\right|}\right) D f_{i j}^{n}\right] .
$$

But, in this way, we unfortunately obtain an unstable algorithm; that is, $f_{i j}^{n+1}$ is not bounded by the same bounds of $f^{n}$. So, to overcome this difficulty, we must replace (6.2) by

$$
\lambda f_{i j}^{n+1}-\operatorname{div}\left(D f_{i j}^{n+1}\right)=p_{i j}-\operatorname{div}\left[\left(1-\frac{\phi^{\prime}\left(\left|D f_{i j}^{n}\right|\right)}{\left|D f_{i j}^{n}\right|}\right) D f_{i j}^{n+1}\right],
$$

which is equivalent to

$$
\lambda f_{i j}^{n+1}-\operatorname{div}\left(\frac{\phi^{\prime}\left(\left|D f_{i j}^{n}\right|\right)}{\left|D f_{i j}^{n}\right|} D f_{i j}^{n+1}\right)=p_{i j},
$$

i.e., (6.1) after discretization.

We multiply (6.1) by $h^{2}$ and we denote by $c_{1}\left(f_{i j}^{n}\right), c_{2}\left(f_{i j}^{n}\right), c_{3}\left(f_{i j}^{n}\right)$, and $c_{4}\left(f_{i j}^{n}\right)$ in (6.1), the coefficients of $f_{i+1, j}^{n+1}, f_{i-1, j}^{n+1}, f_{i, j+1}^{n+1}$, and $f_{i, j-1}^{n+1}$, respectively. With these notations, (6.1) can be written as

$$
\begin{aligned}
& \left(\lambda h^{2}+c_{1}\left(f_{i j}^{n}\right)+c_{2}\left(f_{i j}^{n}\right)+c_{3}\left(f_{i j}^{n}\right)+c_{4}\left(f_{i j}^{n}\right)\right) f_{i j}^{n+1} \\
& =c_{1}\left(f_{i j}^{n}\right) f_{i+1, j}^{n+1}+c_{2}\left(f_{i j}^{n}\right) f_{i-1, j}^{n+1}+c_{3}\left(f_{i j}^{n}\right) f_{i, j+1}^{n+1}+c_{4}\left(f_{i j}^{n}\right) f_{i, j-1}^{n+1}+\lambda h^{2} p_{i j} .
\end{aligned}
$$

We remark that $c_{i} \geq 0$, for $i=1,4$. Now, for $f_{i j}^{n}$, let $C_{i}\left(f_{i j}^{n}\right)$ and $C\left(f_{i j}^{n}\right)$ be defined by

$$
C_{i}=\frac{c_{i}}{\lambda h^{2}+c_{1}+c_{2}+c_{3}+c_{4}}, \quad C=\frac{\lambda h^{2}}{\lambda h^{2}+c_{1}+c_{2}+c_{3}+c_{4}} .
$$


Then, we have that $C_{i}, C \geq 0$ and $C_{1}+C_{2}+C_{3}+C_{4}+C=1$ (we recall that these coefficients depend on $f_{i j}^{n}$ ).

Hence, we write (6.3) as

(6.4) $f_{i j}^{n+1}=C_{1}\left(f_{i j}^{n}\right) f_{i+1, j}^{n+1}+C_{2}\left(f_{i j}^{n}\right) f_{i-1, j}^{n+1}+C_{3}\left(f_{i j}^{n}\right) f_{i, j+1}^{n+1}+C_{4}\left(f_{i j}^{n}\right) f_{i, j-1}^{n+1}+C\left(f_{i j}^{n}\right) p_{i j}$.

Now let $(E,\|\cdot\|)$ be the Banach space

$$
E=\left\{f=\left(f_{i j}\right)_{i, j=1, N}, f_{i j} \in \mathbb{R}\right\} \text { with }\|f\|=\sup _{i j}\left|f_{i j}\right|,
$$

and the subspace $M \subset E: M=\left\{f \in E ; m_{1} \leq f_{i j} \leq m_{2}\right\}$.

PROPOSITION 6.1.

i) If $f^{n} \in M$, then there exists a unique $f^{n+1} \in E$ such that (6.3) is satisfied. Moreover, $f^{n+1} \in M$.

ii) The nonlinear discrete problem

$$
f_{i j}=C_{1}\left(f_{i j}\right) f_{i+1, j}+C_{2}\left(f_{i j}\right) f_{i-1, j}+C_{3}\left(f_{i j}\right) f_{i, j+1}+C_{4}\left(f_{i j}\right) f_{i, j-1}+C\left(f_{i j}\right) p_{i j}
$$

has a solution $f \in M$.

Proof.

i) For $u \in M$, we define the linear application $Q_{u}: M \rightarrow E$ by

$\left(Q_{u}(z)\right)_{i j}=C_{1}\left(u_{i j}\right) z_{i+1, j}+C_{2}\left(u_{i j}\right) z_{i-1, j}+C_{3}\left(u_{i j}\right) z_{i, j+1}+C_{4}\left(u_{i j}\right) z_{i, j-1}+C\left(u_{i j}\right) p_{i j}$.

We will easily prove that $Q_{u}(M) \subset M$ and, moreover, that $Q_{u}$ is a contractive function on $E$. We have, for $z \in M$,

$$
\begin{aligned}
\left(Q_{u}(z)\right)_{i j} & =C_{1}\left(u_{i j}\right) z_{i+1, j}+C_{2}\left(u_{i j}\right) z_{i-1, j}+C_{3}\left(u_{i j}\right) z_{i, j+1}+C_{4}\left(u_{i j}\right) z_{i, j-1} \\
& +C\left(u_{i j}\right) p_{i j} \leq\left(C_{1}\left(u_{i j}\right)+C_{2}\left(u_{i j}\right)+C_{3}\left(u_{i j}\right)+C_{4}\left(u_{i j}\right)+C\left(u_{i j}\right)\right) m_{2}=m_{2} .
\end{aligned}
$$

We obtain in the same way that $m_{1} \leq\left(Q_{u}(z)\right)_{i j}$. Hence, $Q_{u}(z) \in M$. For $v, w \in E$, we have

$$
\begin{aligned}
\left|\left(Q_{u}(v)-Q_{u}(w)\right)_{i j}\right| & \leq C_{1}\left(u_{i j}\right)\left|v_{i+1, j}-w_{i+1, j}\right|+C_{2}\left(u_{i j}\right)\left|v_{i-1, j}-w_{i-1, j}\right| \\
& +C_{3}\left(u_{i j}\right)\left|v_{i, j+1}-w_{i, j+1}\right|+C_{4}\left(u_{i j}\right)\left|v_{i, j-1}-w_{i, j-1}\right| \\
& \leq\left(C_{1}\left(u_{i j}\right)+\cdots+C_{4}\left(u_{i j}\right)\right)\|v-w\| \leq c\|v-w\|,
\end{aligned}
$$

where the positive constant $c$ is

$$
c=\frac{4 \sup _{[0, \infty[} \psi}{\lambda h^{2}+4 \sup _{[0, \infty[} \psi}<1,
$$

since the function $\psi$ is bounded. So, by the classical Banach fixed point theorem, we deduce that there is a unique $f^{n+1} \in E$ such that $f^{n+1}=Q_{f^{n}}\left(f^{n+1}\right)$, which is the fixed point of $Q_{f^{n}}$, or the solution of (6.3). Moreover, $f^{n+1} \in M$.

ii) To prove ii), we define the application $F: M \rightarrow M$ by $F(u)=u^{*}$, where $u^{*}$ is the unique fixed point of $Q_{u}$. We will prove that this application is continuous from the compact and convex set $M \rightarrow M$, and then we will have the existence of a fixed point of $F$, which will be a solution of (6.4).

So, let $u_{n}, u \in M$ such that $\lim _{n \rightarrow \infty}\left\|u_{n}-u\right\|=0$ and $u_{n}^{*}=F\left(u_{n}\right), u^{*}=F(u)$. We have the following equalities and inequalities:

$$
\begin{aligned}
\left\|u_{n}^{*}-u^{*}\right\|=\left\|Q_{u_{n}}\left(u_{n}^{*}\right)-Q_{u}\left(u^{*}\right)\right\| & =\left\|Q_{u}\left(u_{n}^{*}\right)-Q_{u}\left(u^{*}\right)+Q_{u_{n}}\left(u_{n}^{*}\right)-Q_{u}\left(u_{n}^{*}\right)\right\| \\
& \leq\left\|Q_{u}\left(u_{n}^{*}\right)-Q_{u}\left(u^{*}\right)\right\|+\left\|Q_{u_{n}}\left(u_{n}^{*}\right)-Q_{u}\left(u_{n}^{*}\right)\right\| \\
& \leq c\left\|u_{n}^{*}-u^{*}\right\|+\left\|Q_{u_{n}}\left(u_{n}^{*}\right)-Q_{u}\left(u_{n}^{*}\right)\right\| .
\end{aligned}
$$


Then, we get the following:

$$
\begin{array}{r}
(1-c)\left\|u_{n}^{*}-u^{*}\right\| \leq\left\|Q_{u_{n}}\left(u_{n}^{*}\right)-Q_{u}\left(u_{n}^{*}\right)\right\| \leq\left\|u_{n}^{*}\right\| \sup _{i j}\left(\left|C_{1}\left(u_{n i j}\right)-C_{1}\left(u_{i j}\right)\right|+\cdots\right. \\
\left.+\left|C_{4}\left(u_{n i j}\right)-C_{4}\left(u_{i j}\right)\right|+\left|C\left(u_{n i j}\right)-C\left(u_{i j}\right)\right|\right) .
\end{array}
$$

Now, since $\left\|u_{n}^{*}\right\| \leq m_{2}$, for all $n>0$ and since the functions $C_{i}, C$ are continuous (because the functions $\psi$ and minmod are continuous), we obtain that the right-hand side of the least inequality converges to 0 for $n \rightarrow \infty$. Hence $\left\|u_{n}^{*}-u^{*}\right\| \rightarrow 0$; that is, the application $F$ is continuous.

Remarks.

(1) The conclusion i) of Proposition 6.1 says that the algorithm is unconditionally stable. Moreover, to compute $f^{n+1}$ as a solution of the linear system (6.4), since $Q_{f^{n}}$ is contractive, we can use the iterative method

$$
f_{0} \in M, \quad f_{k+1}=Q_{f^{n}}\left(f_{k}\right) \text { and } \lim _{k \rightarrow \infty} f_{k}=f^{n+1} .
$$

Finally, in practice, to accelerate the convergence to the solution $f$ of (6.4), by a combination of these two iterative methods, we use a scheme based on the GaussSeidel algorithm: for $i, j=1,2, \ldots, N$ in this order, we let

$$
f_{i j}^{n+1}=C_{1} f_{i+1, j}^{n}+C_{2} f_{i-1, j}^{n+1}+C_{3} f_{i, j+1}^{n}+C_{4} f_{i, j-1}^{n+1}+C p_{i j},
$$

where for the computation of $C_{1}, \ldots, C_{4}$ and $C$, we replace, respectively, $f_{i-1, j}^{n}, f_{i, j-1}^{n}$ by $f_{i-1, j}^{n+1}, f_{i, j-1}^{n+1}$.

Hence, in practice, we observe that the algorithm is quite stable and convergent.

(2) The conclusion ii) of Proposition 6.1 says that the problem (6.4), which is a nonlinear discrete problem associated with (E), has a solution $f$. In the convex case, we also have the uniqueness of this solution. But we have not proved the convergence of $f^{n}$ to $f$ (in fact, if $f^{n}$ converges, which is true in practice, then this will converge to the solution $f$ of the nonlinear discrete problem).

Now, we will briefly treat the case $R \neq I$. In many cases, the degradation operator $R$, the blur, is a convolution-type integral operator.

In the numerical approximations, $\left(R_{m n}\right)_{m, n=0, d}$ is a symmetric matrix with

$$
\sum_{m, n=0}^{d} R_{m n}=1
$$

and the approximation of $R f$ can be

$$
R f_{i j}=\sum_{m, n=1}^{d} R_{m n} f_{i+\frac{d}{2}-m, j+\frac{d}{2}-n} .
$$

Since $R$ is symmetric, then $R^{*}=R$ and $R^{*} R f=R R f$ is approximated by

$$
R^{*} R f_{i j}=\sum_{m, n=1}^{d} \sum_{r, t=1}^{d} R_{m n} R_{r t} f_{i+d-r-m, j+d-t-n} .
$$

Then, we use the same approximation of the divergence term and the same iterative algorithm, with a slight modification: let

$$
\begin{array}{r}
\lambda h^{2} R^{*} R f_{i j}^{n+1}+\left(c_{1}\left(f_{i j}^{n}\right)+c_{2}\left(f_{i j}^{n}\right)+c_{3}\left(f_{i j}^{n}\right)+c_{4}\left(f_{i j}^{n}\right)\right) f_{i j}^{n+1} \\
=c_{1}\left(f_{i j}^{n}\right) f_{i+1, j}^{n+1}+c_{2}\left(f_{i j}^{n}\right) f_{i-1, j}^{n+1}+c_{3}\left(f_{i j}^{n}\right) f_{i, j+1}^{n+1}+c_{4}\left(f_{i j}^{n}\right) f_{i, j-1}^{n+1}+\lambda h^{2} R p_{i j} .
\end{array}
$$



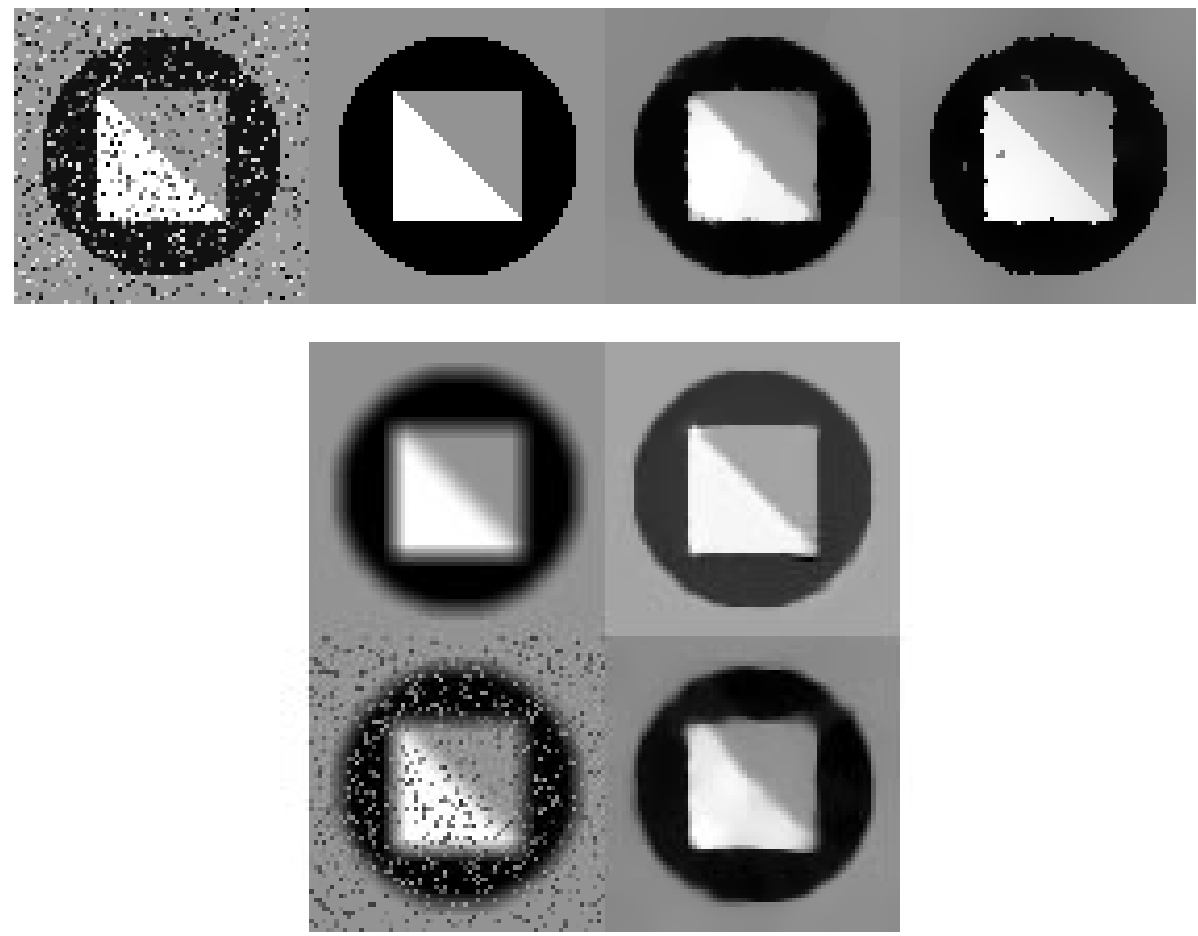

FIG. 7.1. The first sequence of images represents, in the denoising case, from left to right: the degraded image, the synthetic image before degradation, and the reconstructed images with $\phi_{1}$ and $\phi_{2}$. The second represents, from left to right: the degraded image and the reconstructed image; from top to bottom, the deblurring case and both denoising and deblurring, with $\phi_{1}$.

Now, to compute $f^{n+1}$ as the solution of this linear system, we can use, for example, the relaxation method [see 7].

Remark. In these algorithms, there are two parameters, $\lambda$ and $h$. We denote $\lambda^{\prime}=\lambda h^{2}$. For the moment, there are not any rigorous choices for the values of $\lambda^{\prime}$ and $h$. But, in practice, we have observed that (as is natural), by decreasing $h$, the edges are better preserved and also, by decreasing $\lambda^{\prime}$, we diffuse the image.

7. Experimental results. Finally, we present some numerical results on two images of varying difficulty. To generate the images, we have used the software Megawave from CEREMADE, at the University of Paris-Dauphine. The first image is a synthetic picture $(71 \times 71$ pixels) with geometric features (like circles, lines, squares $)$. The second is a real image $(256 \times 256$ pixels $)$ representing a photograph of an office. We have introduced in these pictures the types of degradation considered here: standard noise, Gaussian blur (the atmospheric turbulence blur type) or both, and we have made the choice of the parameters $\lambda^{\prime}$ and $h$ in order to increase the signal to noise ratio. We remark that in the denoising case, we obtain the results very fast (in just three iterations), and we obtain good results in the deblurring case. If the degradation involves both noise and blur, the choice of the parameters is more difficult, because we must take a small $\lambda^{\prime}$ in order to obtain a denoising image but, in the same time, $\lambda^{\prime}$ must be large to deblur the image. The results for the synthetic image are all represented in Figure 7.1. 


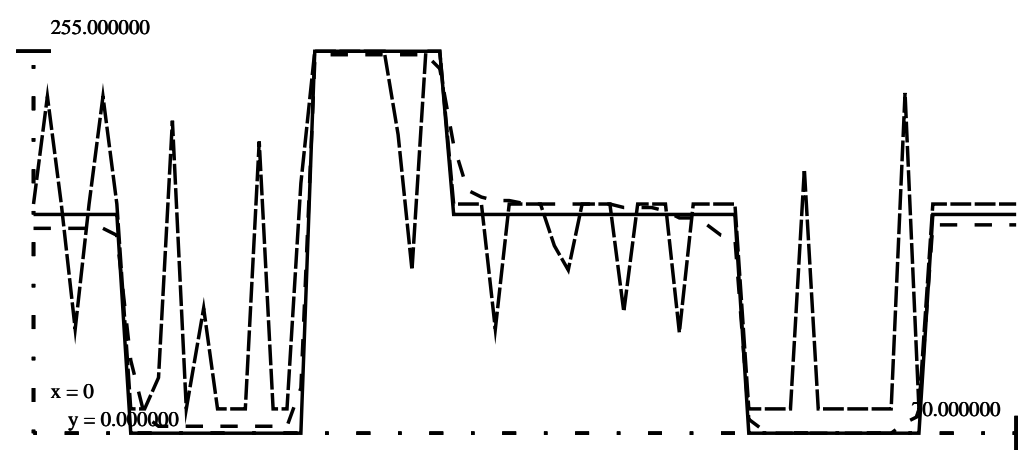

(a)

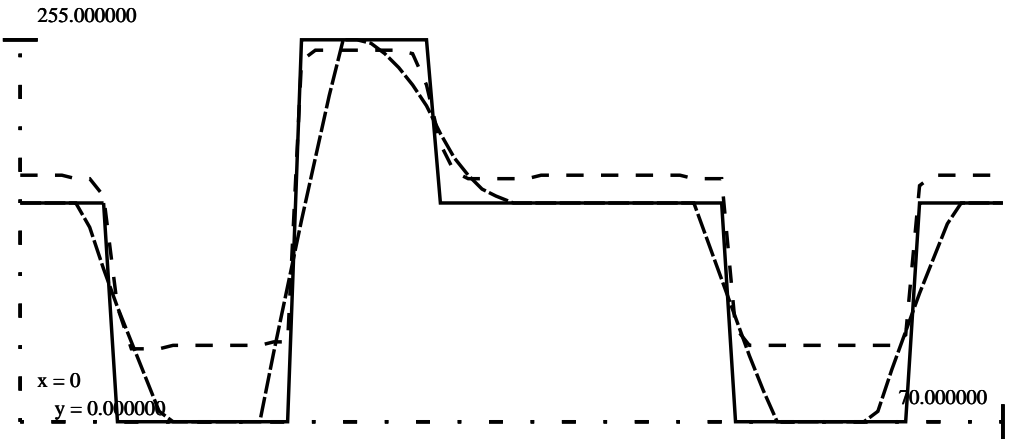

(b)

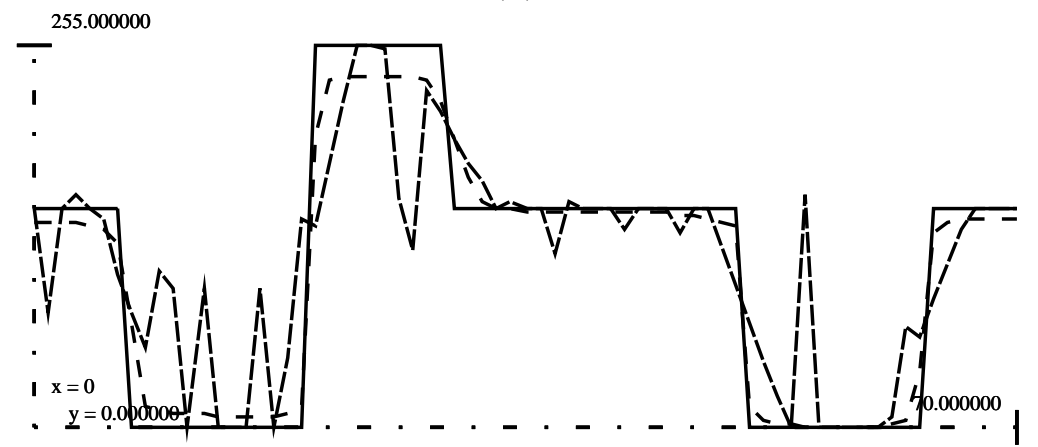

(c)

FIG. 7.2. Profiles of the synthetic image (-), the noisy initial image (- - -), and the reconstructed image (- - -), with the function $\phi_{1}$. We represent in (a) the denoising case and in (b) the deblurring case; (c) involves both noise and blur.

To better illustrate the reconstruction, we have represented in Figure 7.2 the profiles on lines for the first image corresponding to Figure 7.1 (like one-dimensional signals), for each experiment. So, we have superposed the noisy signal, the result, and the signal before degradation.

Finally, in Figure 7.3, we present the results for the real image, which is a picture of an office, in the denoising case and the deblurring case.

Appendix A. In this appendix, we will present the technical lemma of singular perturbations due to Temam [12], which we have used to obtain the a priori estimates 


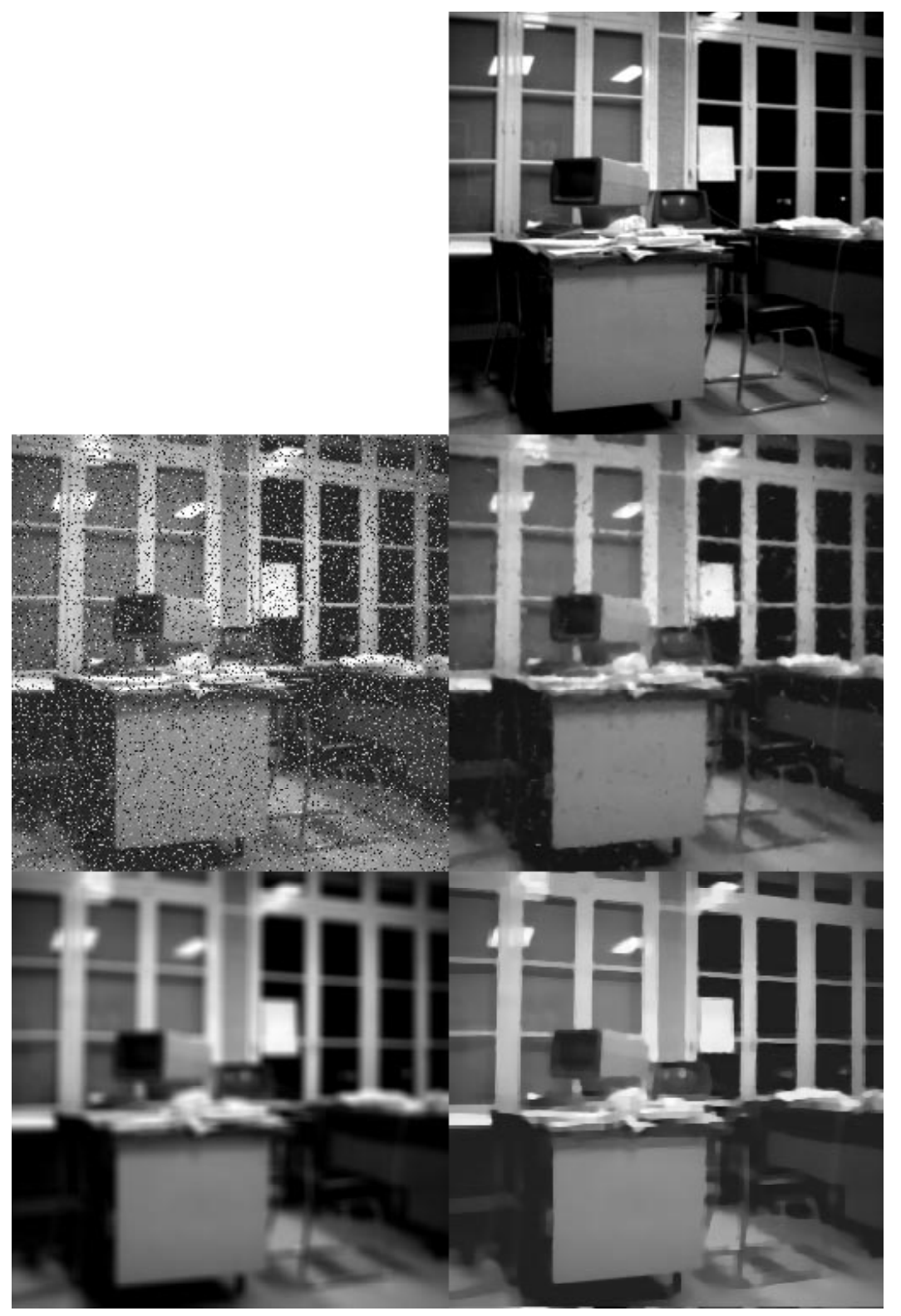

FIG. 7.3. The office picture. From left to right: the degraded image and the reconstructed image. From top to bottom: the denoising case and the deblurring case, with $\phi_{1}$.

in Proposition 4.7. We will state this lemma in an adjusted version of our problem. The goal is to find some estimates independent of $\varepsilon$, on the solution of the problem

$$
\inf _{v \in H^{1}(\Omega)}\left\{\int_{\Omega}(g(D v)+h(v)+p(x) v) d x+\frac{\varepsilon}{2} \int_{\Omega}|D v|^{2} d x\right\} .
$$

We assume the following:

(A.1) the function $\xi \rightarrow g(\xi)$ is convex and of class $C^{3}$ from $\mathbb{R}^{2}$ to $\mathbb{R}$, (A.2) the function $x \rightarrow g(q(x))$ is measurable on $\Omega$, for all $q \in L^{1}(\Omega)^{2}$, 
(A.3) there exist constants $\mu_{i} \geq 0, i=0,8$, such that, for all $\xi \in \mathbb{R}^{2}$,

$$
\begin{gathered}
g(\xi) \geq \mu_{0}|\xi|-\mu_{1}, \mu_{0}>0, \\
\frac{\partial g}{\partial \xi_{i}}(\xi) \leq \mu_{2}, i=1,2, \\
\sum_{i=1}^{2} \frac{\partial g}{\partial \xi_{i}}(\xi) \xi_{i} \geq \mu_{3}\left(1+|\xi|^{2}\right)^{\frac{1}{2}}-\mu_{4}, \mu_{3}>0, \\
\frac{\mu_{6}\left|\eta^{\prime}\right|^{2}}{\left(1+|\xi|^{2}\right)^{\frac{1}{2}}} \leq \sum_{i, j} \frac{\partial^{2} g}{\partial \xi_{i} \partial \xi_{j}}(\xi) \eta_{i} \eta_{j} \leq \frac{\mu_{7}\left|\eta^{\prime}\right|^{2}}{\left(1+|\xi|^{2}\right)^{\frac{1}{2}}} \forall \eta \in \mathbb{R}^{2}, \mu_{6}, \mu_{7}>0,
\end{gathered}
$$

where $\left|\eta^{\prime}\right|^{2}=|\eta|^{2}-\frac{(\eta \cdot \xi)^{2}}{1+|\xi|^{2}}$,

$$
\begin{aligned}
& \qquad\|p\|_{W^{1, \infty}(\Omega)} \leq \mu_{8}, \\
& \sum_{i=1}^{2} \frac{\partial g}{\partial \xi_{i}}(\xi) \xi_{i} \geq 0 \forall \xi \in \mathbb{R}^{2}, \\
& \text { the function } t \rightarrow h(t) \text { is convex and } h^{\prime}(0)=0 .
\end{aligned}
$$

Lemma A.1 (Temam [12]). The problem $\mathcal{P}_{\varepsilon}$ has a unique regular solution $u_{\varepsilon}$ bounded independently of $\varepsilon$ in $L^{\infty}(\Omega) \cap W^{1,1}(\Omega)$. Moreover, for any relatively compact open set $\mathcal{O}$ in $\Omega$, there is a constant $K(\mathcal{O}, \Omega)$ such that

$$
\begin{aligned}
\left\|u_{\varepsilon}\right\|_{W^{1, \infty}(\mathcal{O})} & \leq K, \\
\left\|u_{\varepsilon}\right\|_{H^{2}(\mathcal{O})} & \leq K .
\end{aligned}
$$

We can apply this lemma to our problem by taking, in Proposition 4.7,

$$
g(\xi)=\phi(|\xi|) \text { and } h(t)=t^{2} .
$$

We will not give the proof of this lemma. We refer the reader to the paper and the very technical proofs of Temam. We simply recall that the idea (due to Bernstein) is to obtain some fine estimates on the function $v_{\varepsilon}=\left|D u_{\varepsilon}\right|^{2}$. To do this, we use the Euler equation associated with $\left(\mathcal{P}_{\varepsilon}\right)$, which can be written as

$$
-\varepsilon \triangle u_{\varepsilon}-\sum_{i=1}^{2} \frac{\partial}{\partial x_{i}}\left(\frac{\partial g}{\partial \xi_{i}}\left(D u_{\varepsilon}\right)\right)=-h^{\prime}\left(u_{\varepsilon}\right)-p(x) .
$$

We derive $\left(\mathrm{E}_{\varepsilon}\right)$ with respect to $x_{l}$; then we multiply the result by $\frac{\partial u_{\varepsilon}}{\partial x_{l}}$ and we add over $l$, with $1 \leq l \leq 2$, to get

$$
\begin{aligned}
& \frac{\varepsilon}{2} \sum_{j=1}^{2} \frac{\partial}{\partial x_{j}}\left(\frac{\partial v_{\varepsilon}}{\partial x_{j}}\right)+\varepsilon \sum_{j=1}^{2} \sum_{l=1}^{2}\left(\frac{\partial^{2} u_{\varepsilon}}{\partial x_{l} \partial x_{j}}\right)^{2}-\frac{1}{2} \sum_{i=1}^{2} \frac{\partial}{\partial x_{i}}\left(\sum_{j=1}^{2} \frac{\partial^{2} g}{\partial \xi_{i} \partial \xi_{j}} \frac{\partial v_{\varepsilon}}{\partial x_{j}}\right) \\
& +\sum_{l=1}^{2} \sum_{i=1}^{2} \sum_{j=1}^{2} \frac{\partial^{2} g}{\partial \xi_{i} \partial \xi_{j}} \frac{\partial^{2} u_{\varepsilon}}{\partial x_{l} \partial x_{j}} \frac{\partial^{2} u_{\varepsilon}}{\partial x_{l} \partial x_{i}}=-h^{\prime \prime}\left(u_{\varepsilon}\right) v_{\varepsilon}-\sum_{l=1}^{2} \frac{\partial p}{\partial x_{l}} \frac{\partial u_{\varepsilon}}{\partial x_{l}} .
\end{aligned}
$$

The equation $\left(B_{\varepsilon}\right)$ allows us to obtain the estimates on $v_{\varepsilon}$ by using the test functions judiciously selected and the complicated but classical techniques of Ladyzenskaya and Uralceva [17]. To conclude, we remark that the assumption (A.5) was not given 
by Temam. The Temam assumptions on the integrand dependence in $v$ do not allow us to directly apply its result.

To overcome this difficulty, we have assumed (A.5), and then the term $h^{\prime \prime}\left(u_{\varepsilon}\right) v_{\varepsilon}$ in $\left(B_{\varepsilon}\right)$ is not negative, which allows us to obtain all the a priori estimates proved by Temam.

Appendix B. In the previous sections we studied the problem of image reconstruction when the operator $R=I$ (corresponding to a denoising problem). If $R \neq I$ (generally a convolution operator), the existence and uniqueness results of section 4 remain true if $R$ satisfies the following hypotheses:

(1) $R$ is a continuous and linear operator on $L^{2}(\Omega)$;

(2) $R$ does not annihilate constant functions.

For the results of section 4 , we must suppose in addition that

(3) $R$ is injective.

We do not reproduce the proofs; instead we leave it to the readers to convince themselves.

Acknowledgments. We would like to thank the referees for useful remarks on the first version of the manuscript.

\section{REFERENCES}

[1] L. Alvarez And L. Mazorra, Signal and image restoration using shock filters and anisotropic diffusion, SIAM J. Numer. Anal., 31(1992), pp. 590-605.

[2] F. Catte, P. L. Lions, J. M. Morel, And T. Coll, Image selective smoothing and edge detection by nonlinear diffusion, SIAM J. Numer. Anal., 29(1992), pp. 182-193.

[3] A. Chambolle and P. L. Lions, Image Recovery via Total Variation Minimization and Related Problems, Numer. Math., Vol. 76, 2(1997), pp. 167-188.

[4] P. Charbonnier, Reconstruction d'image: Regularisation avec prise en compte des discontinuitis, Ph.D. thesis, University of Nice-Sophia Antipolis, 1994.

[5] P. Charbonnier, G. Aubert, L. Blanc-Feraud, and M. Barlaud, Two deterministic half-quadratic regularization algorithms for computed imaging, First IEEE Internat. Conf. on Image Processing, Vol. II, Austin, TX, IEEE, Piscataway, NJ, 1994, pp. 168-172.

[6] P. Charbonnier, L. Blanc-Feraud, G. Aubert, and M. Barlaud, Deterministic edgepreserving regularization in computed imaging, IEEE Trans. Image Processing, 6(1997), pp. $298-311$.

[7] P. G. CiARlet, Introduction à l'analyse numerique matricielle et à l'optimisation, Masson, Paris, 1990.

[8] M. Crandall, H. Ishit, And P. L. Lions, User's guide to viscosity solutions of second order partial differential equations, Bull. Amer. Math. Soc., 27(1992), pp. 1-67.

[9] J. P. CrouzeIX, A relationship between the second derivatives of a convex function and of its conjugate, Math. Programming, 13(1977), pp. 364-365.

[10] B. Dacorogna, Direct Method in the Calculus of Variations, Springer-Verlag, Berlin, 1989.

[11] F. Demengel and R. Temam, Convex functions of a measure and applications, Indiana Univ. Math. J., 33(1984), pp. 673-709.

[12] I. Ekeland and R. Temam, Analyse convexe et problhmes variationnels, Dunod-GauthierVillars, Paris, 1974.

[13] S. Geman and D. Geman, Stochastic relaxation, Gibbs distribution and the bayesian restoration of images, IEEE Trans. Pattern Anal. Machine Intell., 6(1984), pp. 721-741.

[14] D. Geman And C. YAng, Nonlinear Image Recovery with Half-Quadratic Regularization and FFt's, preprint, 1993.

[15] D. Geman and G. Reynolds, Constrained restoration and the recovery of discontinuities, IEEE Trans. Pattern Anal. Machine Intell., 14(1992), pp. 367-383.

[16] D. Gilbarg and N. S. Trudinger, Elliptic Partial Differential Equations of Second Order, Springer-Verlag, Berlin, 1983.

[17] O. A. Ladyzenskaya And N. N. URAlceva, Equations aux dérivées partielles de type elliptique, Dunod, Paris, 1968. 
[18] L. Lazaroaia-Vese, Variational Problems and P.D.E.'s in Image Analysis and Curve Evolution, Ph.D. thesis, University of Nice-Sophia Antipolis, 1996.

[19] J. L. Lions And E. Magenes, Problemes aux limites non homogenes, Dunod, Paris, 1968.

[20] P. Perona AND J. MALIK, Scale-space and edge detection using anisotropic diffusion, IEEE Trans. Pattern Anal. Machine Intell., 12(1990), pp. 629-639.

[21] R. T. Rockafellar, Convex Analysis, Princeton University Press, Princeton, NJ, 1970.

$[22]$ S. Osher And L. Rudin, Total variation based image restoration with free local constraints, Proc. IEEE Internat. Conf. on Image Processing, Vol. I, Austin, TX, IEEE, Piscataway, NJ, 1994, pp. 31-35.

[23] L. Rudin, S. Osher, AND E. FAtemi, Nonlinear total variation based noise removal algorithms, Phys. D, 60(1992), pp. 259-268.

[24] G. Stamppachia, Equations elliptiques du second ordre à coefficients discontinus, Presses de l'Université de Montréal, Canada, 1966.

[25] R. Tahraoui, Regularité de la solution d'un problème variationnel, Ann. Inst. H. Poincaré Analyse Non Linéaire, 9(1992), pp. 51-99.

[26] R. Temam, Solutions généralisées de certaines equations du type hypersurfaces minima, Arch. Rational Mech. Anal., 44(1971), pp. 121-156.

[27] A. N. Tikhonov And V. Y. Arsenin, Solutions of Ill-Posed Problems, Wiley, New York, 1977.

[28] A. Visintin, Strong convergence results related to strict convexity, Comm. Partial Differential Equations, 9(1984), pp. 439-466.

[29] C. R. Vogel and M. E. Oman, Iterative Methods for Total Variation Denoising, preprint, 1994, submitted to IEEE Trans. on Image Processing. 